\title{
RICCI FLOW AND THE HOLONOMY GROUP
}

\author{
BRETt L. KotschWAR
}

\begin{abstract}
We prove that the restricted holonomy group of a complete smooth solution to the Ricci flow of uniformly bounded curvature cannot spontaneously contract in finite time; it follows, then, from an earlier result of Hamilton that the holonomy group is exactly preserved by the equation. In particular, a solution to the Ricci flow may be Kähler or locally reducible (as a product) at $t=T$ if and only if the same is true of $g(t)$ at times $t \leq T$.
\end{abstract}

\section{Introduction}

We consider solutions to the Ricci flow

$$
\frac{\partial}{\partial t} g=-2 \operatorname{Rc}(g)
$$

an evolution equation for a smooth family of Riemannian metrics $\left(M^{n}, g(t)\right)$. A wellknown consequence of Hamilton's strong maximum principle for systems $[\mathbf{H 2}]$ is the following characterization of the image of the curvature operator $\mathrm{Rm}: \wedge^{2} T^{*} M \rightarrow$ $\wedge^{2} T^{*} M$ of a solution to (1.1) when this operator is positive semidefinite.

Theorem (Hamilton). Suppose $g(t)$ is a solution to (1.1) on $M \times[0, T]$ satisfying $\operatorname{Rm}(g(t)) \geq 0$. Then there exists $\delta>0$ such that, for $t \in(0, \delta)$, image $(\operatorname{Rm}(g(t))) \subset$ $\wedge^{2} T^{*} M$ is a smooth subbundle invariant under parallel translation with respect to $g(t)$ and closed under the bracket

$$
[\omega, \eta]_{i j}=g^{k l}\left(\omega_{i k} \eta_{l j}-\omega_{j k} \eta_{l i}\right)
$$

Moreover, for any $0<t_{1}<t_{2} \leq T$, image $\left(\operatorname{Rm}\left(g\left(t_{1}\right)\right)\right) \subset$ image $\left(\operatorname{Rm}\left(g\left(t_{2}\right)\right)\right)$.

The theorem is of particular utility in low dimensions, where there are few possibilities for the subalgebra $\operatorname{Rm}\left(\wedge^{2} T_{p}^{*} M\right) \subset \mathfrak{s o}(n)$. In three dimensions, for example, it implies that such a solution must have $\operatorname{Rm}(g(t))>0$ for $t>0$ or split locally as a metric product. The strict code for membership in the class of of solutions with nonnegative curvature operator may lead one to wonder what possibilities there are for a solution $g(t)$ that attains $\operatorname{Rm}\left(g\left(t_{0}\right)\right) \geq 0$ everywhere only after some elapsed time $t_{0}>0$. The condition $\operatorname{Rm}(g(t)) \geq 0$ will be preserved for $t>t_{0}$, and a solution that splits locally for $t_{0}<t<T$ must likewise split at $t=t_{0}$, but we have no information on the properties of the solution prior to $t_{0}$. In particular, we cannot dismiss the possibility that such a solution could split spontaneously at $t_{0}$. One may wonder, more generally, whether it is possible for any solution (on a manifold with compatible topology) to acquire a novel local metric splitting within finite time. Here, one's intuitive picture of the Ricci flow as a "heat equation" for Riemannian metrics seems at odds with such a phenomenon. Surely it must violate some principle of unique continuation. The basic question this paper seeks to answer is: which one?

The author was supported in part by NSF grant DMS-034540.

Received December 2010. 
Our main result is the following theorem. Here $\operatorname{Hol}^{0}(g(t))$ denotes the reduced holonomy group of $g(t)$.

Theorem 1.1. Suppose $g(t)$ is a smooth complete solution to (1.1) on $M \times[0, T]$ of uniformly bounded curvature. Then $\mathrm{Hol}^{0}(g(t)) \subset \mathrm{Hol}^{0}(g(T))$ for all $0 \leq t \leq T$.

Theorem 1.1 is the "backwards-time" analog of the observation of Hamilton (cf. [H2], [H4]) that the holonomy group of a smooth solution to the Ricci flow cannot expand within its lifetime. Thus one actually has $\operatorname{Hol}^{0}(g(t))=\operatorname{Hol}^{0}(g(0))$ along the flow. One consequence is an affirmation of the expectation above that locally product metrics are, in a sense, rigid within the class of solutions to Ricci flow.

Corollary 1.2. Let $(M, g(t))$ be as in Theorem 1.1. Then $(M, g(T))$ is locally reducible (respectively, Kähler) if and only if $(M, g(t))$ is locally reducible (Kähler) for $0 \leq t<T$.

One can equivalently phrase Theorem 1.1 in terms of the time-invariance of the dimensions of the spaces of $\nabla_{g(t)}$-parallel tensors.

Theorem 1.3. If $(M, g(t))$ is as in Theorem 1.1, and $\eta \in C^{\infty}\left(T_{l}^{k}(M)\right)$ satisfies $\nabla_{g(T)} \eta=0$, then there exists a smooth family $\eta(t) \in C^{\infty}\left(T_{l}^{k}(M)\right)$ for $t \in[0, T]$ such that $\nabla_{g(t)} \eta(t)=0$ and $\eta(T)=\eta$.

Since the reduced holonomy groups $\operatorname{Hol}_{p}^{0}(g(t))$ are connected Lie subgroups of $S O\left(T_{p} M\right) \cong S O(n)$, Theorem 1.1 is equivalent to the following infinitesimal reformulation (with the choice $\mathcal{H}=\mathfrak{h o r}(g(T))$ ).

Theorem 1.4. Let $g(t)$ be a complete solution to (1.1) on $M^{n} \times[0, T]$ with $\sup |\operatorname{Rm}(x, t)| \leq K_{0}$. Suppose there exists a smooth subbundle $\mathcal{H} \subset \wedge^{2} T^{*} M$ that is invariant by $\nabla_{g(T)}$-parallel translation and closed under the bracket $[\cdot, \cdot]_{g(T)}$. Then, if image $(\operatorname{Rm}(g(T))) \subseteq \mathcal{H}$, it follows that image $(\operatorname{Rm}(g(t))) \subseteq \mathcal{H}$ and that $\mathcal{H}$ remains invariant by $\nabla_{g(t)}$-parallel translation and closed under the bracket $[\cdot, \cdot]_{g(t)}$ for all $t \in[0, T]$. Moreover, $\mathfrak{h o l}_{p}(g(t)) \subseteq \mathcal{H}_{p}$ for all $(p, t) \in M \times[0, T]$.

We divide the proof of Theorem 1.4 into several steps. In Section 3, we reduce it to a problem of unique continuation for a certain system; this is Theorem 3.7. In Section 4 we embed this system in a larger (closed) system of coupled partial- and ordinary-differential inequalities. The bulk of the work is the verification that this larger system is indeed closed; for this we must perform a rather careful analysis of the evolution equations of the components of our system. For the unique continuation, we ultimately appeal to a special case of an earlier result of the author $[\mathbf{K}]$ for parabolic PDE-ODE systems. The approach in that reference was inspired by work of Alexakis $[\mathbf{A}]$ on weakly-hyperbolic systems arising in the study of the vacuum Einstein equations.

We remark that, although we restrict our attention to the Ricci flow in the present paper, the basic method can be applied to study the holonomy of families of metrics arising from other geometric evolution equations. For example, a result analogous to Theorem 1.1 holds for the metrics induced by the mean curvature flow of hypersurfaces in Euclidean space (and, with additional conditions, in more general ambient spaces); we intend to address this in a future note.

\section{Motivation: non-expansion of holonomy.}

As we mentioned above, it is a result of Hamilton (cf. [H2], [H4]) that a solution to Ricci flow with holonomy initially restricted to some subgroup of $S O(n)$ will continue to have its holonomy so restricted. For this paper, the statement of this "non-expansion" result we have in mind is the following. 
Theorem 2.1 (Hamilton). Suppose $g(t)$ is a smooth complete solution to (1.1) with $g(0)=g_{0}$ and $|\operatorname{Rm}(g(x, t))| \leq K_{0}$ on $M^{n} \times[0, T]$. If $\operatorname{Hol}^{0}\left(g_{0}\right)=G \subset S O(n)$, we have $\mathrm{Hol}^{0}(g(t)) \subset G$ for $0 \leq t \leq T$.

Theorems 1.1 and 2.1 are statements about the backwards- and forwards-time behavior of a solution to a (weakly-) parabolic system, and, despite their apparent symmetry, require rather different methods of proof. For the purpose of comparison, we will discuss two proofs of Theorem 2.1 in detail. The first is an elementary combination of Berger's classification [Ber], de Rham's splitting theorem [DR], and the uniqueness of solutions for the Ricci flow $[\mathbf{H 1}],[\mathbf{C Z}]$. The second, which we defer to the appendix, is essentially self-contained and closer to the argument suggested in [H4].

We first give an example to show that, in general, one cannot dispense with the restriction that $g(t)$ be complete (cf. also the similar example on p. 247 of $[\mathbf{C L N}]$ ).

Example 2.2 (Flat-sided sphere). Let $U \subset S^{2}$ be a proper open set, $x_{0} \in S^{2} \backslash U$, and $h_{0}$ a metric on $S^{2}$ of Gaussian curvature $K_{h_{0}} \geq 0$ satisfying $K_{h_{0}} \equiv 0$ on $U$ but $K_{h_{0}}\left(x_{0}\right)>0$. One can take, e.g., $x_{0}$ to be the north pole, $U$ a small disk about the south pole and $\phi \in C^{\infty}\left(S^{2},[0,1]\right)$ with $\phi \equiv 1$ on the upper hemisphere and $\phi \equiv 0$ on $U$. By the theorem of Kazdan-Warner $[\mathbf{K W}]$, one can find a metric $h_{0}$ with $K_{h_{0}}=\phi$, and, for this metric, there exists $T>0$ and a solution $h(t)$ to the Ricci flow defined for $t \in[0, T)$ with $h(0)=h_{0}$. For any $a>0$, we can define a solution $g_{a}(t)$ to Ricci flow on $U$ by

$$
g_{a}(x, t) \doteqdot \begin{cases}\left.h_{0}\right|_{U}(x) & (x, t) \in U \times[0, a] \\ h(x, t-a) & (x, t) \in U \times(a, a+T) .\end{cases}
$$

For $0<t \leq a, K_{g_{a}(t)} \equiv 0$, but the strong maximum principle implies $K_{h(t)}>0$ for $t>0$, so $K_{g_{a}(t)}>0$ for $t>a$. Thus $\left(U, g_{a}(t)\right)$ satisfies $\operatorname{Hol}^{0}\left(g_{a}(t)\right)=\{I d\}$ for $t \leq a$, but $\operatorname{Hol}^{0}\left(g_{a}(t)\right)=S O(2)$ for $a<t<T$.

2.1. Non-expansion via Berger's Classification. All of the ingredients of the proof below can be found, for example, in the combination of the references $[\mathbf{H 4}]$ and $[\mathbf{J}]$. The argument can be summarized very succinctly. In the category of complete solutions to the Ricci flow with bounded curvature, any initial isometries are preserved, and product, Kähler, and Einstein initial data extend uniquely to solutions of the same type. With the splitting theorem [DR] and the classification theorem $[\mathbf{B e r}]$ as it is now understood, this is enough to conclude that any restriction of the initial holonomy is shared by the solution at later times.

We will refer to the following modern version of Berger's theorem (cf., e.g., Theorem 3.4.1, $[\mathbf{J}])$.

Theorem 2.3 (Berger). If $M^{n}$ is simply connected and $g$ is irreducible, then either $g$ is symmetric or exactly one of the following hold:

1) $\operatorname{Hol}^{0}(g)=S O(n)$,

2) $n=2 m$ with $m \geq 2$, and $\operatorname{Hol}^{0}(g)=U(m)$ in $S O(2 m)$,

3) $n=2 m$ with $m \geq 2$, and $\operatorname{Hol}^{0}(g)=S U(m)$ in $S O(2 m)$,

4) $n=4 m$ with $m \geq 2$, and $\operatorname{Hol}^{0}(g)=\operatorname{Sp}(m)$ in $S O(4 m)$,

5) $n=4 m$ with $m \geq 2$, and $\operatorname{Hol}^{0}(g)=\mathrm{Sp}(m) \cdot \operatorname{Sp}(1)$ in $S O(4 m)$,

6) $n=7$ and $\mathrm{Hol}^{0}(g)=G_{2}$ in $S O(7)$, or

7) $n=8$ and $\mathrm{Hol}^{0}(g)=\operatorname{Spin}(7)$ in $S O(8)$.

First proof of Theorem 2.1. First, we may assume that $M$ is simply connected, as $\operatorname{Hol}^{0}\left(\tilde{g_{0}}\right)=\operatorname{Hol}^{0}\left(g_{0}\right)$ if $\tilde{g_{0}}$ is the lift of $g_{0}$ to the universal cover of $M$. We may also assume $\mathrm{Hol}^{0}\left(g_{0}\right)$ is irreducible. Otherwise, by de Rham's splitting theorem, $\left(M, g_{0}\right)$ 
splits as a global product

$$
\left(M, g_{0}\right) \cong\left(N_{1} \times N_{2} \times \cdots \times N_{m}, g_{1} \oplus g_{2} \cdots \oplus g_{m}\right) .
$$

Each metric $g_{i}$ will be complete and of bounded curvature $\left|\operatorname{Rm}\left(g_{i}\right)\right| \leq K_{0}$, and so, by the existence theorems of Hamilton $[\mathbf{H 1}]$ and Shi $[\mathbf{S}]$, each factor $N_{i}$ will admit a complete solution $g_{i}(t)$ of bounded curvature with $g_{i}(0)=g_{i}$ on some small time interval $\left[0, T_{i}\right]$ (with $T_{i}$ depending only $K_{0}$ and $\operatorname{dim}\left(N_{i}\right)$ ). Then $\hat{g}(t) \doteqdot g_{1}(t) \oplus$ $g_{2}(t) \cdots \oplus g_{m}(t)$ will be a complete solution of bounded curvature on $M \times[0, \delta]$ for $\delta>0$ equal to the minimum of the $T_{i}$. But, by uniqueness, there is only one solution of bounded curvature with initial data $g_{0}$, hence $g(t) \equiv \hat{g}(t)$ on $M \times[0, \delta]$. The argument may then be iterated on intervals of uniform size to obtain the agreement of $g(t)$ with a product solution on all of $M \times[0, T]$. Since we may then consider each factor independently, we may as well assume that $g_{0}$ is irreducible.

Now we consider each case of Theorem 2.3 in turn. Suppose first that $g_{0}$ is symmetric. The uniqueness of solutions and the diffeomorphism invariance of the equation imply that $\operatorname{Isom}(g(0)) \subset \operatorname{Isom}(g(t))$. For a general metric $g$, denote by $A(g)$ the set of isometries $A(g) \doteqdot\left\{\sigma_{q} \in \operatorname{Iso}_{q}(g) \mid \sigma_{q}^{2}=I d\right\}$. Since the composition law of $\operatorname{Isom}(g(t)) \subset \operatorname{Diff}(M)$ and the set of any isometry's fixed points are independent of the metric, the preservation of initial isometries also implies $A\left(g_{0}\right) \subset A(g(t))$. In particular, $g(t)$ remains symmetric for $t>0$. But for a symmetric metric $g$, each fixed representative $\operatorname{Hol}_{p}^{0}(g)$ of the isomorphism class of $\operatorname{Hol}^{0}(g)$ can be described explicitly as the subgroup of squares of involutive isometries fixing $p$ (cf. Proposition 3.35 of $[\mathbf{J}])$. Symbolically,

$$
\operatorname{Hol}_{p}^{0}(g)=J_{p}(g) \doteqdot \operatorname{Iso}_{p}(g) \cap\left\{\sigma_{q} \circ \sigma_{r} \mid \sigma_{q}, \sigma_{r} \in A(g)\right\} .
$$

Then $A\left(g_{0}\right) \subset A(g(t))$ implies $J_{p}\left(g_{0}\right) \subset J_{p}(g(t))$ and, since $g(t)$ is symmetric, that

$$
\operatorname{Hol}_{p}^{0}(g(t))=J_{p}(g(t)) \subset J_{p}\left(g_{0}\right)=\operatorname{Hol}_{p}\left(g_{0}\right) .
$$

Therefore we are left with the seven alternatives on Berger's list. The first of these is uninteresting, of course, as $\operatorname{Hol}^{0}(g) \subset S O(n)$ for any metric $g$. The second, $\operatorname{Hol}^{0}\left(g_{0}\right)=U(n / 2)$, implies $g_{0}$ is Kähler, and it is well-known that from a Kähler initial metric of bounded curvature one can construct a Kähler solution of bounded curvature by the solution of an appropriate parabolic Monge-Ampere equation for the potential. This solution may, a priori, only exist for a short time, but for this period we must have $g(t) \equiv \hat{g}(t)$ by uniqueness (and hence $\operatorname{Hol}^{0}(g(t)) \subset U(n / 2)$ ). We may then iterate as before to conclude the same on the entire interval of existence for $g(t)$.

This leaves five cases. However, in each of these, $g_{0}$ is necessarily Einstein (cf. pp. 53-55 of $[\mathbf{J}]$ ). (In fact, in the cases $S U(m), \operatorname{Sp}(m), \operatorname{Spin}(7)$, or $G_{2}$, the metric must be Ricci-flat.) But, associated to Einstein initial data $\operatorname{Rc}\left(g_{0}\right)=\rho g_{0}$, one can construct the Einstein solution $\hat{g}(t)=(1-2 \rho t) g_{0}$ which moves only by homothetical scaling. The holonomy is obviously unchanged for this solution and it is unique among (at least) those of uniformly bounded curvature. Thus $\hat{g}(t)=g(t)$ and $G=\operatorname{Hol}^{0}(\hat{g}(t))=$ $\operatorname{Hol}^{0}(g(t))$.

q.e.d.

2.2. Berger's theorem and non-contraction of holonomy. It is natural to ask whether one can fashion an analogous argument along for Theorem 1.1. The answer seems to be "only partially." The failure of this argument to extend to all cases was, in fact, the starting point for the work in the present paper.

Of the three primary components of the preceding proof, we nevertheless retain at least two. The classification component, coming from Berger's and de Rham's theorems and their consequences, is as applicable to $g(T)$ as it was to $g(0)$. From $[\mathbf{K}]$, we also have a counterpart to the uniqueness component: two complete solutions 
$g(t), \tilde{g}(t)$ to (1.1) of uniformly bounded curvature that agree at $t=T>0$ must agree at times $t<T$. From this, it follows that any isometries of $g(T)$ are shared by $g(t)$ for $t<T$, and that $g(T)$ is Einstein only if $g(t)$ is as well for $t<T$.

What we lack, rather, is the ability to construct by hand the special "competitor" solutions to extend the data $g(T)$ to a solution of the same type for times $t<T$. Of course, if $g(T)$ is Einstein, we may still construct an extension by homothetical scaling of $g(T)$. However, when $g(T)$ is Kähler, we cannot simply construct a Kähler extension $\tilde{g}(t)$ for $T-\delta<t \leq T$ by the method above, since we must now specify instead the data for the potential at time $T$. Such "terminal-value" parabolic problems are ill-posed and lack solutions in general. The analogous terminal-value problems for the Ricci (or Ricci - De Turck) flows are also ill-posed, and this is an impediment, in particular, to the construction of a product extension $\tilde{g}(t)=\tilde{g}_{1}(t) \oplus \tilde{g}_{2}(t)$ for $t<T$ from product data $g(T)=g_{1} \oplus g_{2}$ on $N_{1} \times N_{2}$. The trouble is that, while the product metric $g_{1} \oplus g_{2}$ belongs to $R F(M, T)$ - the "image" of the time- $T$ Ricci flow operator on $M$, we do not know whether either of the factors $g_{i}$ belong to $R F\left(N_{i}, \delta\right)$ for any $\delta>0$.

While Theorem 1.1 is not simply reducible to the backwards-uniqueness of solutions to (1.1), we will show, nevertheless, that it is equivalent to the backwardsuniqueness of a certain larger, mixed parabolic and ordinary-differential, system. The argument we will describe in the next section (and carry out in those following) will be essentially self-contained and, in particular, independent of the theorems of Berger and de Rham.

\section{Non-contraction of $\mathrm{Hol}^{0}(g(t))$ as a problem of unique continuation.}

Our basic strategy is to interpret restricted holonomy as a condition on the operator $\mathrm{Rm}: \wedge^{2} T^{*} M \rightarrow \wedge^{2} T^{*} M$. (This is also the basis of Hamilton's approach to non-expansion of holonomy in [H4]). This characterization is natural since the curvature effectively determines the holonomy Lie algebra (in a manner we will review below), but it offers an additional advantage for our purposes in that the curvature operator, unlike the metric, satisfies a strictly parabolic equation.

The representation of the holonomy Lie algebra hol $(g(T))$ on $T M$ gives a subbundle of $\wedge^{2} T^{*} M$ that is invariant under parallel translation and closed under the Lie-bracket given by (1.2). The image of the curvature operator is contained in $\mathfrak{h o l}(g(T))$ and, as $\operatorname{Rm}(g(T))$ is self-adjoint, its kernel at each $p$ therefore contains $\mathfrak{h o l}_{p}(g(T))^{\perp}$. The bundle $\mathfrak{h o l}(g(T))^{\perp}$ is likewise closed under parallel translation, though not in general under the Lie bracket. The following observation shows that (as in Theorem 1.4) we may as well consider any parallel subalgebra $\mathcal{H}$ containing $\operatorname{Rm}(g(T)), \mathfrak{h o l}(g(T))$ being, in a sense, the minimal such $\mathcal{H}$.

Lemma 3.1. Suppose $\mathcal{H} \subset \wedge^{2}\left(T^{*} M\right)$ is a smooth distribution closed under parallel transport and the Lie bracket (1.2). If, for all $p \in M$, image $(\operatorname{Rm}(g(p))) \subset \mathcal{H}_{p}$, then $\mathfrak{h o l}_{p}(g) \subset \mathcal{H}_{p}$.

Proof. This follows easily from the Ambrose-Singer theorem [AS] (cf. also Besse [Bes], Theorem 10.58) which says that the elements of the leftmost union in the chain of inclusions

$$
\bigcup_{q \in M, \gamma \in \Omega_{p, q}, \omega \in \wedge^{2} T_{p}^{*} M}\left(\tau_{\gamma} \circ \operatorname{Rm}(q) \circ \tau_{\gamma}^{-1}\right)(\omega) \subset \bigcup_{q \in M, \gamma \in \Omega_{p, q}} \tau_{\gamma}^{*}\left(\mathcal{H}_{q}\right) \subset \mathcal{H}_{p} .
$$

generate $\mathfrak{h o l}_{p}(g)$. Here $\Omega_{p, q}$ represents the space of piecewise smooth paths $\gamma:[0,1] \rightarrow$ $M$ with $\gamma(0)=p, \gamma(1)=q$ and $\tau_{\gamma}$ represents the extension of parallel transport along the path $\gamma$ to two-forms.

q.e.d. 
Assuming then we have such a $\mathcal{H} \subset \wedge^{2} T^{*} M$, we consider its perpendicular complement $\mathcal{K} \doteqdot \mathcal{H}^{\perp}$ and associated orthogonal projection operator $\widehat{P}_{T}: \wedge^{2} T^{*} M \rightarrow \mathcal{K}$. Although we ultimately wish to show that $\left.\operatorname{Rm}(g(t))\right|_{\mathcal{K}} \equiv 0$, we do not know a priori whether, for $t<T$, the fibers of $\mathcal{H}$ and $\mathcal{K}$ are complementary orthogonal subspaces (or that those of $\mathcal{H}$ are closed under the bracket (1.2)) relative to $g(t)$. Thus we first define time-dependent extensions $H(t)$ and $K(t)$ for $\mathcal{H}$ and $\mathcal{K}$ that retain these properties on $[0, T]$. Then we prove $\left.\operatorname{Rm}(g(t))\right|_{K(t)} \equiv 0$ (hence image $\left.(\operatorname{Rm}(g(t))) \subset H(t)\right)$ and use this to show $H(t) \equiv \mathcal{H}$ and $K(t) \equiv \mathcal{K}$.

We define $H(t)$ and $K(t)$ as the images of the families of projection maps $\bar{P}(t)$ and $\widehat{P}(t)$ extending $\bar{P}_{T}$ and $\widehat{P}_{T}$. We have $\nabla_{g(T)} \bar{P}_{T} \equiv \nabla_{g(T)} \widehat{P}_{T} \equiv 0$ and $\operatorname{Rm}(g(T)) \circ \widehat{P}_{T} \equiv$ 0 , and, by spelling out the mandate that they remain complementary orthogonal projections, it is not hard to determine what these extensions $\bar{P}(t)$ and $\widehat{P}(t)$ ought to be, namely, the solutions to $D_{t} \widehat{P}=0$ on $[0, T]$ with $\bar{P}(T)=\bar{P}_{T}$ and $\widehat{P}(T)=\widehat{P}_{T}$. Here $D_{t}$ represents a time-like vector tangent to the submanifold of $g(t)$-orthonormal frames in the product of the frame bundle with the interval: $F(M) \times[0, T]($ see $(3.1)$ and Section 4.2). This extension, in any event, is achieved by solving an ODE on each fiber of $\wedge^{2} T_{p}^{*} M$. With $\widehat{P}(t)$ so obtained, we arrive at the following "backwardsuniqueness" problem: to show $\operatorname{Rm} \circ \widehat{P}(t) \equiv 0$ and $\nabla \widehat{P}(t) \equiv 0$ for all $0 \leq t<T$, given their vanishing at $t=T$. Once it has been established, all that remains is to verify that $K(t)=$ image $(\widehat{P}(t))$ is in fact constant in time. This is a consequence of the equation satisfied by $\widehat{P}(t)$, and we do this in Lemma 3.6 below.

The remainder of the present section will be dedicated to the reduction of Theorem 1.4 to a precise statement of the backwards-uniqueness problem described above; this will be Theorem 3.7.

3.1. Some preliminaries. The following elementary observation will in fact be essential to the computations in Section 4.

Lemma 3.2. Suppose $V$ is a vector space with an inner product $\langle\cdot, \cdot\rangle$ and a consistent Lie bracket $[\cdot, \cdot]$. If $H \subset V$ is a subalgebra, and $K \doteqdot H^{\perp}$, then $[H, K] \subset K$.

Proof. The assumption of consistency implies that the trilinear map

$$
(X, Y, Z) \mapsto\langle[X, Y], Z\rangle
$$

is fully antisymmetric. Thus, if $h_{1}, h_{2} \in H$ and $k \in K$, we have

$$
\left\langle\left[h_{1}, k\right], h_{2}\right\rangle=-\left\langle\left[h_{1}, h_{2}\right], k\right\rangle=0
$$

as $\left[h_{1}, h_{2}\right] \in H=K^{\perp}$.

q.e.d.

Related to the trilinear form in the above proof is the following operator, which we will need to identify in certain of our computations that follow.

Definition 3.3. Suppose $V$ is a vector space with inner product $\langle\cdot, \cdot\rangle$ and Lie bracket $[\cdot, \cdot]$. Let

$$
\mathcal{T}: \operatorname{End}(V) \times \operatorname{End}(V) \times \operatorname{End}(V) \rightarrow V^{*} \otimes V^{*} \otimes V^{*}
$$

be the operator defined by

$$
\mathcal{T}[A, B, C]\left(v_{1}, v_{2}, v_{3}\right) \doteqdot\left\langle\left[A\left(v_{1}\right), B\left(v_{2}\right)\right], C\left(v_{3}\right)\right\rangle
$$

for $A, B, C \in \operatorname{End}(V), v_{i} \in V$.

For completeness, we include the proof of a few elementary properties of projection maps and parallel translation that we will use in the sequel. 
Lemma 3.4. Suppose $M$ is connected and $\pi: V \rightarrow M$ is a smooth $m$-dimensional vector bundle with connection $D$ and a compatible metric $h$ on its fibers. (We will also use $D$ to represent the induced connection $\left.D: \operatorname{End}(V) \rightarrow T^{*} M \otimes \operatorname{End}(V)\right)$. Let $H \subset V$ be an l-dimensional smooth subbundle, $K=H^{\perp}$, and $\bar{P}: V \rightarrow H$, $\widehat{P}: V \rightarrow K$ the $h$-orthogonal projections onto $H$ and $K$.

1) For any $X \in T M$,

$$
\bar{P} \circ D_{X} \bar{P} \circ \bar{P}=\widehat{P} \circ D_{X} \bar{P} \circ \widehat{P}=\bar{P} \circ D_{X} \widehat{P} \circ \bar{P}=\widehat{P} \circ D_{X} \widehat{P} \circ \widehat{P}=0 .
$$

2) The following are equivalent: $H$ is closed under parallel translation, $K$ is closed under parallel translation, $D \bar{P} \equiv 0$, and $D \widehat{P} \equiv 0$.

Proof. For the first claim, we fix $X \in T M$, and differentiate both sides of the identity $\bar{P} \circ \bar{P}=\bar{P}$ to obtain

$$
D_{X} \bar{P} \circ \bar{P}+\bar{P} \circ D_{X} \bar{P}=D_{X} \bar{P} \text {. }
$$

Pre- and post-composing both sides of this result with $\bar{P}$ and using again the above identity, we arrive at

$$
2 \bar{P} \circ D_{X} \bar{P} \circ \bar{P}=\bar{P} \circ D_{X} \bar{P} \circ \bar{P},
$$

from which we conclude $\bar{P} \circ D_{X} \bar{P} \circ \bar{P}=0$. For the second equality in (1), we differentiate both sides of $\widehat{P} \circ \bar{P}=0$ to obtain

$$
D_{X} \widehat{P} \circ \bar{P}+\widehat{P} \circ D_{X} \bar{P}=0 \text {. }
$$

If we now pre- and post-compose both sides with $\widehat{P}$, the first term on the left vanishes, and we are left with $\widehat{P} \circ D_{X} \bar{P} \circ \widehat{P}=0$. The identities for $D_{X} \widehat{P}$ follow similarly.

For the second claim, first note that, since $\bar{P}+\widehat{P}=\mathrm{Id}: V \rightarrow V$, we have $D \bar{P}=0$ if and only if $D \widehat{P}=0$. Suppose now that $D \bar{P}=D \widehat{P}=0$. Given $p, q \in M, X \in H_{p}$ and $\gamma:[0,1] \rightarrow M$ a smooth curve joining $p$ to $q$, define $X(t) \in V_{\gamma(t)}$ by parallel transport along $\gamma$. If $T=\gamma_{*}\left(\frac{d}{d t}\right)$, then $D_{T} \widehat{P}=0$ and $D_{T} X=0$ along $\gamma$. But the compatibility of the metric with $D$ implies $f(t)=|\widehat{P}(X(t))|_{h}^{2}$ satisfies

$$
f^{\prime}(t)=2\left\langle D_{T} \widehat{P}(X)+\widehat{P}\left(D_{T}(X)(t)\right), \widehat{P}(X(t))\right\rangle=0
$$

and $f(0)=0$. Thus $f \equiv 0$ and, in particular, $\widehat{P}(X(1))=0$, i.e., $X(1) \in K_{q}^{\perp}=$ $H_{q}$. So $H$ is closed under parallel translation. Similarly, $K$ is closed under parallel translation.

Suppose then that, on the other hand, $H$ is invariant under parallel translation. Let $p, q \in M, \gamma:[0,1] \rightarrow M$ a smooth path connecting $p$ and $q,\left\{V_{i}\right\}_{i=1}^{l}$ and $\left\{V_{i}\right\}_{i=l+1}^{m}$ be orthogonal orthogonal bases for $H_{p}$ and $K_{p}$ respectively and $V_{i}(t)$ the parallel transports of $V_{i}$ along $\gamma$. Then $V_{i}(t) \in H_{\gamma(t)}$ for $t \in[0,1]$. For any $i, j$, define $A_{i j}(t)=h(\gamma(t))\left(V_{i}(t), V_{j}(t)\right)$. Then $A_{i j}^{\prime}=0$ as above. Since $A_{i j}(0)=\delta_{i j}$, we have $A_{i j}(t)=\delta_{i j}$. In particular, $H_{q}=\operatorname{span}\left\{V_{i}(1)\right\}_{i=1}^{l}=\left(\operatorname{span}\left\{V_{i}(1)\right\}_{i=l+1}^{m}\right)^{\perp}$, so $K_{q}=\operatorname{span}\left\{V_{i}(1)\right\}_{i=l+1}^{m}$. Since $p$ and $q$ were arbitrary, $K$ is also invariant under parallel translation; obviously we can also reverse the roles of $H$ and $K$.

Finally, suppose again that $H$ (hence, now, also $K$ ) is invariant under parallel translation. We wish to show that $D \bar{P} \equiv 0$ (which is equivalent to $D \widehat{P} \equiv 0$ as remarked above), and for this it suffices to show that $h(p)\left(D_{X} \bar{P}(U), W\right)=0$ for an arbitrary $p \in M, X \in T_{p} M$, and $U, W \in V_{p}$. So let $\gamma:(-\epsilon, \epsilon) \rightarrow M$ be any smooth curve with $\gamma(0)=p$ and $\gamma^{\prime}(0)=X$. Define $U(t)$ and $W(t)$ to be the parallel transports of $U$ and $W$ along $\gamma$ and let $k(t)=h(\gamma(t))(\bar{P} U(t), W(t))$. By the first part of this lemma, we only need to check the "off-diagonal" components of $D_{X} \bar{P}$, that is, the cases in which $U$ and $W$ belong to opposite summands of $V_{p}=H_{p} \oplus K_{p}$. So 
suppose first that $U \in H_{p}$ and $W \in K_{p}$. Since $H$ and $K$ are invariant under parallel translation, $U(t) \in H_{\gamma(t)}$ and $W(t) \in K_{\gamma(t)}$, thus

$$
k(t)=h(\gamma(t))(U(t), W(t)) \equiv h(p)(U, W)=0 .
$$

Similarly, if $U \in K_{p}$ and $W \in H_{p}$, then $\bar{P} U(t) \equiv 0$ and $k(t) \equiv 0$. In both cases, we have $k^{\prime}(0)=h(p)\left(D_{X} U, W\right)=0$.

q.e.d.

3.2. A time-dependent family of distributions. Going forward, let $g(t)$ be a smooth solution of (1.1) on $M^{n}$ for $t \in[0, T]$ and define $h \doteqdot g(T)$. Given a tensor field $V \in T_{l}^{k}(M)$ that is, in some sense, "calibrated" to the metric $g$ at $t_{0} \in[0, T]$, there is a natural (and well-known) means of extending $V$ to a family of sections $V(t)$ for $t \in[0, T]$ with the promise of preserving this calibration. Namely, one can define $V(p, t)$ in each fiber $T_{l}^{k}\left(T_{p} M\right)$ as the solution of the ODE

$$
\begin{aligned}
\frac{\partial}{\partial t} V_{b_{1} b_{2} \cdots b_{l}}^{a_{1} a_{2} \cdots a_{k}}= & R_{c}^{a_{1}} V_{b_{1} b_{2} \cdots b_{l}}^{c a_{2} \cdots a_{k}}+R_{c}^{a_{2}} V_{b_{1} b_{2} \cdots b_{l}}^{a_{1} c \cdots a_{k}}+\cdots+R_{c}^{a_{k}} V_{b_{1} b_{2} \cdots b_{l}}^{a_{1} a_{2} \cdots c} \\
& -R_{b_{1}}^{c} V_{c b_{2} \cdots b_{l}}^{a_{1} a_{2} \cdots a_{k}}-R_{b_{2}}^{c} V_{b_{1} c \cdots b_{l}}^{a_{1} a_{2} \cdots a_{k}}-\cdots-R_{b_{l}}^{c} V_{b_{1} b_{2} \cdots c}^{a_{1} a_{2} \cdots a_{k}}
\end{aligned}
$$

for $t \in[0, T]$ with $V\left(p, t_{0}\right)=V(p)$. Note that if $V=g\left(t_{0}\right)$, this procedure simply recovers the solution $g(t)$, and if $V$ and $W$ are related by an identification of $T M$ with $T M^{*}$ according to the metric $g\left(t_{0}\right)$ (i.e., by raising or lowering indices), then $V(t)$ and $W(t)$ will be related by the analogous identification of $T M$ and $T^{*} M$ according to $g(t)$ on $[0, T]$. Likewise, a contraction of $V$ by $g\left(t_{0}\right)$ evolved according to (3.1) will be the same contraction of $V(t)$ by $g(t)$. Equation (3.1) is equivalent to considering the evaluation of the fixed tensor $V$ on a time-dependent local frame evolved so as to preserve the pairwise inner products of the elements of the frame. We will consider a somewhat more formal variation of this identification in the next section; in the notation presented there, the above procedure is equivalent to finding a representative $V$ satisfying $D_{t} V \equiv 0$.

At present, though, (3.1) allows us to identify the distributions $\mathcal{H}$ and $\mathcal{K}$ with convenient relatives $H(t)$ and $K(t)$. We let $\bar{P}_{T}, \widehat{P}_{T} \in \operatorname{End}\left(\wedge^{2} T^{*} M\right)$ denote, respectively, the orthogonal projections onto $\mathcal{H}$ and $\mathcal{K}$ with respect to $h$, and construct $\bar{P}(t)$ and $\widehat{P}(t)$ according to the procedure (3.1) with $\bar{P}(T)=\bar{P}_{T}$ and $\widehat{P}(T)=\widehat{P}_{T}$. Thus, in components, and here regarded as elements of $\operatorname{End}\left(\wedge^{2} T^{*} M\right) \cong T_{4}(M)$ (see Section $4.1)$,

$$
\begin{aligned}
& \frac{\partial}{\partial t} \bar{P}_{a b c d}=-R_{a p} \bar{P}_{p b c d}-R_{b p} \bar{P}_{a p c d}-R_{c p} \bar{P}_{a b p d}-R_{d p} \bar{P}_{a b c p} \\
& \frac{\partial}{\partial t} \widehat{P}_{a b c d}=-R_{a p} \widehat{P}_{p b c d}-R_{b p} \widehat{P}_{a p c d}-R_{c p} \widehat{P}_{a b p d}-R_{d p} \widehat{P}_{a b c p} .
\end{aligned}
$$

We then define

$$
H(t) \doteqdot \operatorname{image}(\bar{P}(t)) \subset \wedge^{2} T^{*} M, \quad \text { and } \quad K(t) \doteqdot \operatorname{image}(\widehat{P}(t)) \subset \wedge^{2} T^{*} M .
$$

We collect here the properties of these subspaces we will need in the sequel.

Lemma 3.5. Let $g, h=g(T), \mathcal{H}, \mathcal{K}, H(t)$, and $K(t)$ be defined as above, and $\operatorname{dim} \mathcal{H}=k$. For all $t \in[0, T], \operatorname{dim} H(t)=k, H(t)$ is closed under the Lie bracket $(1.2)$ with respect to $g(t)$, and $K(t)=H(t)^{\perp}$. Moreover, if $\mathcal{T}$ is defined as in Definition 3.3, we have

$$
\mathcal{T}[\widehat{P}, \bar{P}, \bar{P}]=\mathcal{T}[\bar{P}, \widehat{P}, \bar{P}]=\mathcal{T}[\bar{P}, \bar{P}, \widehat{P}]=0 .
$$

Proof. The first three properties are easily verified from equations (1.2) and (3.1). The last follows then from Lemma 3.2.

We now show that if it happens that image $(\operatorname{Rm}(g(t))) \subset H(t)$ for all $t$, then $H(t)$ and $K(t)$ are actually independent of time. 
Lemma 3.6. With $g(t), H(t), K(t)$ as above, suppose image $(\operatorname{Rm}(g(t))) \subset H(t)$ for all $t \in[0, T]$. Then $H(t) \equiv H(T)=\mathcal{H}, K(t) \equiv K(T)=\mathcal{K}$.

Proof. Let $m=n(n-1) / 2$ and $p \in M$, and $h=g(p, T)$. Choose an find an $h$-orthonormal basis $\left\{\varphi^{A}\right\}_{A=1}^{m}$ of sections for $\wedge^{2} T_{p}^{*} M$ such that $\left\{\varphi^{A}\right\}_{A=1}^{k}$ is a basis for $\mathcal{H}_{p}$ and $\left\{\varphi^{A}\right\}_{A=k+1}^{m}$ a basis for $\mathcal{K}_{p}$. We can then use the procedure described by equation (3.1) on the individual forms $\varphi^{A}$ to a produce a family of two-forms $\left\{\varphi^{A}(t)\right\}_{A=1}^{m}$ on $T_{p} M$ for $t \in[0, T]$. This set will be a $g(t)$-orthonormal basis for $\wedge^{2} T_{p}^{*} M$ for any $t$, and moreover,

$$
\bar{P}(t)\left(\varphi^{A}(t)\right)= \begin{cases}\varphi^{A}(t) & \text { if } \quad A \leq k \\ 0 & \text { if } \quad A>k\end{cases}
$$

and

$$
\widehat{P}(t)\left(\varphi^{A}(t)\right)=\left\{\begin{array}{lll}
0 & \text { if } \quad A \leq k \\
\varphi^{A}(t) & \text { if } \quad A>k
\end{array}\right.
$$

Thus $\left\{\varphi^{A}(t)\right\}_{A=1}^{k}$ and $\left\{\varphi^{B}(t)\right\}_{A=k+1}^{m}$ remain bases for $H(t)$ and $K(t)$, respectively. In fact, for $t \in[0, T]$,

$$
\bar{P}(t)=\sum_{A=1}^{k}\left(\varphi^{A}(t)\right)^{*} \otimes \varphi^{A}(t), \quad \widehat{P}(t)=\sum_{A=k+1}^{m}\left(\varphi^{A}(t)\right)^{*} \otimes \varphi^{A}(t) .
$$

Now, for any fixed $t$, we can choose an orthonormal basis $\left\{e_{a}\right\}$ of $T_{p} M$ relative to $g(p, t)$; in these components $g_{a b}(p, t)=\delta_{a b}$. Let $M$ be the symmetric matrix defined by

$$
R_{a b c d}=-M_{A B} \varphi_{a b}^{A} \varphi_{c d}^{B} .
$$

For any $A$, at $(p, t)$ we have (observing the extended summation condition),

$$
\begin{aligned}
\frac{\partial}{\partial t} \varphi_{a b}^{A} & =-R_{a q} \varphi_{q b}^{A}-R_{b q} \varphi_{a q}^{A} \\
& =-R_{a p p q} \varphi_{q b}^{A}-R_{b p p q} \varphi_{a q}^{A} \\
& =M_{B C}\left(\varphi_{a p}^{B} \varphi_{p q}^{C} \varphi_{q b}^{A}+\varphi_{b p}^{B} \varphi_{p q}^{C} \varphi_{a q}^{A}\right) \\
& =M_{B C}\left(\left(\varphi_{a p}^{B}\left[\varphi^{C}, \varphi^{A}\right]_{p b}+\varphi_{a p}^{B} \varphi_{b q}^{C} \varphi_{q p}^{A}\right)+\left(\varphi_{b p}^{B}\left[\varphi^{C}, \varphi^{A}\right]_{a p}+\varphi_{b p}^{B} \varphi_{a q}^{C} \varphi_{p q}^{A}\right)\right) \\
& =M_{B C}\left[\left[\varphi^{A}, \varphi^{C}\right], \varphi^{B}\right]_{a b}-R_{a p b q} \varphi_{q p}^{A}-R_{b p a q} \varphi_{p q}^{A} \\
& =M_{B C}\left[\left[\varphi^{A}, \varphi^{C}\right], \varphi^{B}\right]_{a b}+\left(R_{a p b q}+R_{p b a q}\right) \varphi_{q p}^{A} \\
& =M_{B C}\left[\left[\varphi^{A}, \varphi^{C}\right], \varphi^{B}\right]_{a b}-R_{b a p q} \varphi_{q p}^{A} \\
& =M_{B C}\left[\left[\varphi^{A}, \varphi^{C}\right], \varphi^{B}\right]_{a b}+\operatorname{Rm}\left(\varphi^{A}\right)_{a b},
\end{aligned}
$$

where the penultimate line follows from the Bianchi identity. That is,

$$
\frac{\partial}{\partial t} \varphi^{A}=L\left(\varphi^{A}\right)+\operatorname{Rm}\left(\varphi^{A}\right)
$$

where $L: \wedge^{2} T_{p}^{*} M \rightarrow \wedge^{2} T_{p}^{*} M$ is the linear map determined by

$$
L\left(\varphi^{A}\right)=M_{B C}\left[\left[\varphi^{A}, \varphi^{C}\right], \varphi^{B}\right] .
$$

Note that (3.3) is independent of the frame $\left\{e_{a}\right\}$.

We claim that $L$ satisfies $L\left(H_{p}(t)\right) \subset K_{p}(t)$ and $L\left(K_{p}(t)\right) \subset H_{p}(t)$. First, since image $(\operatorname{Rm}(p, t)) \subset H_{p}(t)$, and $\mathrm{Rm}$ is symmetric, it follows that $K_{p}(t) \subset$ $\operatorname{ker}(\operatorname{Rm}(p, t))$, and hence that $M_{B C}=0$ if $B>k$ or $C>k$. Also, by Lemmas 
3.2 and 3.5 , we have $\left[H_{p}(t), H_{p}(t)\right] \subseteq H_{p}(t)$, and $\left[H_{p}(t), K_{p}(t)\right] \subseteq K_{p}(t)$. Stated in terms of the structure constants

$$
C_{C}^{A B}=\left\langle\left[\varphi^{A}, \varphi^{B}\right], \varphi^{C}\right\rangle
$$

this is $C_{C}^{A B}=0$ if $A, B \leq k$ and $C>k$, or exactly one of $A$ and $B$ is greater than $k$ and $C \leq k$. Now,

$$
L\left(\varphi^{A}\right)=\sum_{B, C \leq k} \sum_{1 \leq D, E \leq m} M_{B C} C_{D}^{A C} C_{E}^{D B} \varphi^{E} .
$$

If $A \leq k$, then each $C_{D}^{A C}$ is only nonzero for $D \leq k$,

$$
L\left(\varphi^{A}\right)=\sum_{B, C, D, E \leq k} M_{B C} C_{D}^{A C} C_{E}^{D B} \varphi^{E} \doteqdot \sum_{1 \leq E \leq k} S_{E}^{A} \varphi^{E} .
$$

Likewise, if $A>k$, the only non-zero occurences of $C_{D}^{A C}$ in (3.4) are those with $D>k$, thus restricting the non-zero occurences of $C_{E}^{D B}$ in the sum to those with $E>k$. So

$$
L\left(\varphi^{A}\right)=\sum_{B, C \leq k} \sum_{k<D, E} M_{B C} C_{D}^{A C} C_{E}^{D B} \varphi^{E} \doteqdot \sum_{k<E \leq m} T_{E}^{A} \varphi^{E}
$$

Thus defining

$$
V(t) \doteqdot\left(\varphi^{1}(t), \varphi^{2}(t), \ldots, \varphi^{k}(t)\right)^{T}, \quad W(t) \doteqdot\left(\varphi^{k+1}(t), \varphi^{k+2}(t), \ldots, \varphi^{m}(t)\right)^{T},
$$

and using that $\operatorname{Rm}\left(\varphi^{A}\right) \equiv 0$ if $A>k$, we can restate (3.3) as a matrix equation

$$
\left(\begin{array}{c}
\dot{V}(t) \\
\dot{W}(t)
\end{array}\right)=\left(\begin{array}{cc}
S(t)+M(t) & 0 \\
0 & T(t)
\end{array}\right)\left(\begin{array}{c}
V(t) \\
W(t)
\end{array}\right) .
$$

It follows, then, that for all $0 \leq t \leq T$, for appropriate coefficients $E_{B}^{A}(t)$,

$$
\varphi^{A}(t)=\sum_{B=1}^{k} E_{B}^{A}(t) \varphi^{B}(T) \in H(T)=\mathcal{H}
$$

if $A \leq k$. Similarly, if $A>k$, we have $\varphi^{A}(t) \in \mathcal{K}$ for all $0 \leq t \leq T$.

q.e.d.

3.3. A restatement of Theorem 1.4. Now we are able to frame Theorem 1.4 as a problem of unique continuation. Under the assumptions of that theorem, we have, by Lemma 3.5, a $g(t)$-orthogonal decomposition $\wedge^{2} T^{*} M=H(t) \oplus K(t)$ where $H(t)$ remains closed under the Lie bracket. By the symmetry of the operator Rm, we will have image $(\operatorname{Rm}(t)) \subset H(t)$ if and only if $K(t) \subset \operatorname{ker}(\operatorname{Rm}(t))$, i.e., if $\operatorname{Rm} \circ \widehat{P}(t) \equiv 0$ for all $t \in[0, T]$. But if image $(\operatorname{Rm}(t)) \subset H(t)$, it follows from Lemma 3.6 that $H(t) \equiv \mathcal{H}$ and $K(t) \equiv \mathcal{K}$. To conclude from Lemma 3.1 that $\mathfrak{h o l}_{p}(g(t)) \subset \mathcal{H}_{p}$, we need to know further that $H(t)=\mathcal{H}$ is closed under parallel translation with respect to $\nabla_{g(t)}$ for all $t$. However, by Lemma 3.4, this is true if and only if $\nabla \widehat{P} \equiv 0$ on $M \times[0, T]$. Therefore, Theorem 1.4 is a consequence of the following assertion.

Theorem 3.7. Under the assumptions of Theorem 1.4, we have

$$
\operatorname{Rm} \circ \widehat{P} \equiv 0, \quad \nabla \widehat{P} \equiv 0
$$

on $M \times[0, T]$ where $\widehat{P}=\widehat{P}(t)$ is the projection onto $K(t)$ with respect to $g(t)$ in the orthogonal decomposition $\wedge^{2} T^{*} M=H(t) \oplus K(t)$ provided by Lemma 3.5.

We remark that, given the dependence of the evolutions of $\nabla_{g(t)}$ and $\widehat{P}(t)$ on the curvature, the aims of proving $\operatorname{Rm} \circ \widehat{P} \equiv 0$ and $\nabla \widehat{P} \equiv 0$ are not independent. We will establish them simultaneously in the course of proving Theorem 3.7. 


\section{A PDE-ODE System}

A few back-of-the-envelope calculations should convince the reader that the system consisting of $\widehat{R} \doteqdot \mathrm{Rm} \circ \widehat{P}$ and $\nabla \widehat{P}$ is neither parabolic nor too far from being so. First, it is easy to see that the application of the heat operator to $\widehat{R}$ produces a term involving unmatched second derivatives of $\widehat{P}$. Schematically,

$$
\left(D_{t}-\Delta\right) \widehat{R}=\left(\left(D_{t}-\Delta\right) \mathrm{Rm}\right) * \widehat{P}+\nabla \mathrm{Rm} * \nabla \widehat{P}+\mathrm{Rm} * \Delta \widehat{P},
$$

where we use $V * W$ to denote some linear combination of contractions of the tensors $V$ and $W$ by the metric. Since we have only defined $\widehat{P}$ by the means of the fiberwise ODE $D_{t} \widehat{P}=0$, we cannot expect to have much control over $\nabla^{(k)} \widehat{P}$ (beyond observations on the level of (1) of Lemma 3.4). A natural option is to try to adjoin $\nabla \nabla \widehat{P}$ itself to the system. This addition is logically redundant from the perspective of Theorem 3.7 since $\nabla \widehat{P}$ will be parallel on any time-slice on which $\widehat{P}$ is parallel, but it comes at the cost of introducing higher order curvature terms. This can be seen from (3.1) and the standard formula

$$
\frac{\partial}{\partial t} \Gamma_{i j}^{k}=-g^{m k}\left(\nabla_{i} R_{j m}+\nabla_{j} R_{i m}-\nabla_{m} R_{i j}\right),
$$

for the evolution of the Christoffel symbols, which yield

$$
\frac{\partial}{\partial t} \nabla \nabla \widehat{P}=\nabla \nabla \mathrm{Rm} * \widehat{P}+\nabla \mathrm{Rm} * \nabla \widehat{P}
$$

At minimum, we must introduce a component involving $\nabla \mathrm{Rm}$ to our system to compensate (as it turns out, and unlike the second derivatives of $\widehat{P}$, the factors of $\nabla \nabla \mathrm{Rm}$ may be controlled by regarding them, effectively, as factors of $\nabla(\nabla \mathrm{Rm}))$. From the perspective of Theorem 3.7, the correct (i.e., redundant) such component ought to be $\widehat{T} \doteqdot \nabla \operatorname{Rm} \circ(\operatorname{Id} \times \widehat{P})$, that is, the element of $T M^{*} \otimes \operatorname{End}\left(\wedge^{2} T^{*} M\right)$ given by

$$
\widehat{T}(X, \omega) \doteqdot\left(\nabla_{X} \operatorname{Rm}\right)(\widehat{P}(\omega)),
$$

since it must also vanish on any time slice where $\widehat{R} \equiv 0$ and $\nabla \widehat{P} \equiv 0$. (In fact, for any $X$, the images of the endomorphisms $\nabla_{X} \operatorname{Rm}(g(p))$ lie in $\mathfrak{h o l}_{p}(g)$, cf. Remark 10.60 of $[$ Bes $]$.) Fortunately, with this addition, our system stabilizes. The tensor $\nabla \mathrm{Rm}$ satisfies a heat-type equation with reaction terms containing only products and contractions of $\mathrm{Rm}$ and $\nabla \mathrm{Rm}$ :

$$
\left(\frac{\partial}{\partial t}-\Delta\right) \nabla \mathrm{Rm}=\nabla \mathrm{Rm} * \mathrm{Rm}
$$

and the Laplacian falling on the composition $\widehat{T}$ generates only contractions of firstand second-covariant derivatives of $\widehat{P}$ with $\nabla \mathrm{Rm}$ and $\nabla \nabla \mathrm{Rm}$. Thus we see that the application of the heat operator to $\widehat{T}$ introduces no fundamentally new quantities. While we have been rather cavalier about the manner in which the components of the terms are combined (relative to the decomposition $H(t) \oplus K(t)$ ), we nevertheless are entitled to some optimism that the collection of $\widehat{R}, \widehat{T}, \nabla \widehat{P}$, and $\nabla \nabla \widehat{P}$ will fit into a closed system of mixed differential inequalities. We will use the rest of this section to make this heuristic argument precise.

4.1. Notation and statement. In this section, we assume we have a solution to Ricci flow $g(t)$ and distributions $\mathcal{H}$ and $\mathcal{K}$ as in Theorem 1.4. Let $H(t)$ and $K(t)$ be the distributions described in Lemma 3.5, and $\bar{P}(t), \widehat{P}(t)$ their associated projections. We fix notation, once and for all, for the following collection of tensors:

$$
\begin{array}{lll}
\bar{R} \doteqdot \mathrm{Rm} \circ \bar{P}, & \widehat{R} \doteqdot \mathrm{Rm} \circ \widehat{P}, \\
\bar{T} \doteqdot \nabla \mathrm{Rm} \circ(I d \times \bar{P}), & \widehat{T} \doteqdot \nabla \mathrm{Rm} \circ(I d \times \widehat{P}), \\
A \doteqdot \nabla \widehat{P}, & B & \doteqdot \nabla \nabla \widehat{P} .
\end{array}
$$


Note that $\bar{P}$ and $\widehat{P}$ are self-adjoint elements of $E \doteqdot \operatorname{End}\left(\wedge^{2} T^{*} M\right)$. It will be convenient to use the metric identification of $E \cong\left(\wedge^{2} T^{*} M\right)^{*} \otimes \wedge^{2} T^{*} M$ with $\wedge^{2} T^{*} M \otimes \wedge^{2} T^{*} M$ and further with the subspace of $T_{4}(M)$ in which the members are antisymmetric in the first two and last two arguments. We make this identification by selecting the normalization

$$
V \wedge W=\frac{1}{2}(V \otimes W-W \otimes V)
$$

for $V, W$ in $T M$ (or $T^{*} M$ ) With respect to a local frame $\left\{e_{a}\right\}$ for $T^{*} M$, we have

$$
\bar{P}_{a b c d}=\left\langle\bar{P}\left(e_{a} \wedge e_{b}\right), e_{c} \wedge e_{d}\right\rangle, \quad \widehat{P}_{a b c d}=\left\langle\widehat{P}\left(e_{a} \wedge e_{b}\right), e_{c} \wedge e_{d}\right\rangle,
$$

so, if $\omega \in \wedge^{2} T^{*} M$,

$$
\bar{P}(\omega)_{c d}=\bar{P}_{a b c d} \omega_{a b}, \quad \widehat{P}(\omega)_{c d}=\widehat{P}_{a b c d} \omega_{a b} .
$$

We also define $T_{m a b c d}=\nabla_{m} R_{a b c d}$.

However, for the endomorphism Rm, since we wish to keep the notation $R_{a b c d}$ consistent with the usual convention (namely, that with respect to which one has $\langle\operatorname{Rm}(\omega), \omega\rangle \geq 0$ and $R_{a b b a} \geq 0$ on the standard sphere), we have an additional minus sign in our formula:

$$
\operatorname{Rm}(\omega)_{c d}=-R_{a b c d} \omega_{a b}=R_{a b d c} \omega_{a b}
$$

Similarly,

$$
\left(\nabla_{X} \mathrm{Rm}\right)(\omega)_{c d}=-\nabla_{m} R_{a b c d} X_{m} \omega_{a b}=T_{m a b d c} X_{m} \omega_{a b} .
$$

The tensors $\bar{P}$ and $\widehat{P}$, like $R$, are symmetric in the interchange of their first and last pairs of indices and antisymmetric in the interchange of the elements of those pairs:

$$
\begin{aligned}
\bar{P}_{a b c d} & =\bar{P}_{c d a b}=-\bar{P}_{a b d c}=-\bar{P}_{b a c d}, \\
\widehat{P}_{a b c d} & =\widehat{P}_{c d a b}=-\widehat{P}_{a b d c}=-\widehat{P}_{b a c d} .
\end{aligned}
$$

We also have $A_{\text {mabcd }}=\nabla_{m} \widehat{P}_{a b c d}, B_{\text {mnabcd }}=\nabla_{m} \nabla_{n} \widehat{P}_{a b c d}$, for which corresponding identities hold. The tensors $R$ and $T$ are of course, also subject to the Bianchi identities.

The tensors $\bar{R}, \widehat{R}, \bar{T}, \widehat{T}$ are no longer symmetric in the interchange of the final two pairs of indices, but remain antisymmetric in the interchange of the elements of these pairs:

$$
\begin{aligned}
\widehat{R}_{i j k l} & =\widehat{P}_{i j a b} R_{a b l k}=-\widehat{R}_{j i k l}=-\widehat{R}_{i j l k}, \\
\widehat{T}_{m i j k l} & =\widehat{P}_{i j a b} T_{m a b l k}=-\widehat{T}_{m j i k l}=-\widehat{T}_{m i j l k},
\end{aligned}
$$

and similarly for $\bar{R}$ and $\bar{T}$.

Now, we let $E \doteqdot \operatorname{End}\left(\wedge^{2} T^{*} M\right)$,

$$
\begin{aligned}
& \mathcal{X} \doteqdot E \oplus\left(T^{*} M \otimes E\right) \cong T_{4}(M) \oplus T_{5}(M), \\
& \mathcal{Y} \doteqdot\left(T^{*} M \otimes E\right) \oplus\left(T^{*} M \otimes T^{*} M \otimes E\right) \cong T_{5}(M) \oplus T_{6}(M),
\end{aligned}
$$

and define

$$
\mathbf{X}(t) \doteqdot \widehat{R}(t) \oplus \widehat{T}(t) \in \mathcal{X}
$$

and

$$
\mathbf{Y}(t) \doteqdot A(t) \oplus B(t) \in \mathcal{Y} .
$$

The goal of this section is to prove the following result. 
Proposition 4.1. With the above definitions, and under the assumptions of Theorem 1.4, for all $\delta>0$, there exists a $C=C\left(n, K_{0}, \delta, T\right)$ such that on $M \times[\delta, T]$, we have

$$
\begin{aligned}
\left|\left(\frac{\partial}{\partial t}-\Delta_{g(t)}\right) \mathbf{X}\right|_{g(t)}^{2} & \leq C\left(|\mathbf{X}|_{g(t)}^{2}+|\mathbf{Y}|_{g(t)}^{2}\right) \\
\left|\frac{\partial}{\partial t} \mathbf{Y}\right|_{g(t)}^{2} & \leq C\left(|\mathbf{X}|_{g(t)}^{2}+|\nabla \mathbf{X}|_{g(t)}^{2}+|\mathbf{Y}|_{g(t)}^{2}\right)
\end{aligned}
$$

Here we use the same notation to denote the metrics on $\mathcal{X}, \mathcal{Y}$, and $T M^{*} \otimes \mathcal{X}$ induced by $g(t)$, and $\nabla=\nabla_{g(t)}$ and $\Delta_{g(t)}$ to denote the connection and Laplacian induced on $\mathcal{X}$ by $g(t)$ and its Levi-Civita connection.

REMARK 4.2. The parameter $\delta$ is an artifact of what will be an eventual application of Shi's estimates $[\mathbf{S}]$ for the derivatives of the curvature tensor, reflecting the degradation of the estimates as $t \rightarrow 0$. If $M$ is compact, one can dispense with $\delta$ in favor of an estimate valid for all $t \in[0, T]$, but with a constant $C$ that now also depends on the suprema of the norms of the first and second derivatives of curvature on $M \times[0, T]$.

4.2. The orthonormal frame bundle associated to $g(t)$. The verification of (4.3) and (4.4) will depend closely on the algebraic structure of the evolution equations $\widehat{R}, \widehat{T}, A, B$. To aid the computations, we will regard the tensors as functions on the product of the $g(t)$-orthonormal frame bundle $\mathcal{O}(M)$ with the interval $[0, T]$. The utility of this perspective to calculations attached to the study of Ricci flow was first demonstrated by Hamilton in [H3]. For our application, we will borrow the notation and abide by the conventions of Appendix F of [CCG2], thus, in particular, some commutation formulas involving curvature will differ by a sign from their counterparts in $[\mathbf{H 3}]$.

Following [CCG2], we let $\pi: F(M) \rightarrow M$ denote the frame bundle of $M$. This is a principal $G L(n, \mathbb{R})$-bundle on $M$; we take the group to act on the left. On $\mathfrak{g l}(n, \mathbb{R})$, one has the standard basis of elements $\{e(a, b)\}_{a, b=1}^{n}$, with $e(a, b)_{c}^{d}=\delta_{c}^{a} \delta_{b}^{d}$. We may fix a metric $h$ on $\mathfrak{g l}(n, \mathbb{R})$ by insisting on the orthonormality of this basis with respect to $h$. Thus,

$$
\langle e(a, b), e(c, d)\rangle_{h}=\delta_{d}^{a} \delta_{b}^{c} .
$$

Let $\mu: G L(n, \mathbb{R}) \times F(M) \rightarrow F(M)$ denote the left action and, for any frame $Y$, define $\mu_{Y}: G L(n, \mathbb{R}) \rightarrow F(M)$ by $\mu_{Y}(A) \doteqdot \mu(A, Y)$. Then we have the isomorphism $\left(\mu_{Y}\right)_{*}: \mathfrak{g l}(n, \mathbb{R}) \rightarrow T_{Y}\left(F(M)_{x}\right)$ defining the vertical spaces $\mathcal{V}_{Y}=\operatorname{image}\left(\left(\mu_{Y}\right)_{*}\right)$, where $\pi(Y)=x$. At each $Y$, the Levi-Civita connection $\nabla$ of $g(t)$ defines complementary horizontal spaces $\mathcal{W}_{Y} \subset T_{Y}(F(M))$. For each $t \in[0, T]$, there is a unique metric $g^{F}(t)$ on $F(M)$ which enforces the orthogonality of the subbundles $\mathcal{V}$ and $\mathcal{W}$ and for which $\pi:\left(F(M), g^{F}(t)\right) \rightarrow(M, g(t))$ is a submersion and

$$
\left(\mu_{Y}\right)_{*}:(\mathfrak{g l}(n, \mathbb{R}), h) \rightarrow\left(T_{Y}\left(F(M)_{x}\right),\left.g^{F}(t)\right|_{T_{Y}\left(F(M)_{x}\right)}\right)
$$

an isometry at each $Y \in F(M)$.

A solution $g(t)$ to Ricci flow on $M \times(0, T)$ defines a map

$$
\left.g: F(M) \times[0, T] \rightarrow S M_{n}(\mathbb{R})\right)
$$

with values in the symmetric $n \times n$ matrices. Likewise, a time-dependent family of sections of $T_{l}^{k}(M)$ may be regarded as an $O(n)$-equivariant matrix-valued function on $\widetilde{F(M)} \doteqdot F(M) \times[0, T]$. These functions are determined by their values on the 
submanifold

$$
\widetilde{\mathcal{O}(M)} \doteqdot g^{-1}(\mathrm{Id})=\bigcup_{t \in[0, T]} \mathcal{O}(M)_{g(t)} \times\{t\} \subset \widehat{F(M)}
$$

where $\mathcal{O}(M)_{g(t)} \subset F(M)$ denotes the bundle of $g(t)$-orthogonal frames. It is convenient to use the same notation for the tensors under both of these interpretations. Thus for $T \in T_{2}^{1}(M)$, we will write

$$
T_{a b}^{c}=T(Y)_{a b}^{c}=T_{a b}^{c}(x)=T(x)\left(Y_{a}, Y_{b}, Y^{c}\right)
$$

at a given $Y \in F(M)$, where, again, $\pi(Y)=x$, and $Y^{c} \in T_{x}^{*} M$ is the $c$-th element of the frame dual to $Y$ at $x$.

4.3. Elements of a global frame on $T \widetilde{F(M)}$ and their commutators. We continue to follow Appendix F of [CCG2]. From the isomorphisms $\left(\mu_{Y}\right)_{*}: \mathfrak{g l}(n, \mathbb{R}) \rightarrow$ $T_{Y} F(M)_{\pi(Y)}$, we may generate a basis for each $\mathcal{V}_{Y}$ from $\{e(a, b)\}_{a, b=1}^{n}$ by defining, for each $1 \leq a, b \leq n$ and $Y \in F(M)$

$$
\Lambda_{b}^{a}(Y) \doteqdot\left(\mu_{Y}\right)_{*} e(a, b) .
$$

The action of this vector field on a tensor is algebraic. On $U \in T_{2}(M)$, for example, it is given by

$$
\Lambda_{b}^{a} U_{i j}=\delta_{i}^{a} U_{b j}+\delta_{j}^{a} U_{i b}
$$

and on general $U \in T_{l}^{k}(M)$ by

$$
\begin{aligned}
\Lambda_{b}^{a} U_{i_{1} i_{2} \ldots i_{l}}^{j_{1} j_{2} \ldots j_{k}}= & \delta_{i_{1}}^{a} U_{b i_{2} \ldots i_{l}}^{j_{1} j_{2} \ldots j_{k}}+\delta_{i_{2}}^{a} U_{i_{1} b \ldots j_{l}}^{j_{1} j_{2} \ldots j_{k}}+\cdots+\delta_{i_{l}}^{a} U_{i_{1} i_{2} \ldots b}^{j_{1} j_{2} \ldots j_{k}} \\
& -\delta_{b}^{j_{1}} U_{i_{1} i_{2} \ldots j_{l}}^{a j_{2} \ldots j_{k}}-\delta_{b}^{j_{2}} U_{i_{1} i_{2} \ldots i_{l}}^{j_{1} a \ldots j_{k}}-\ldots-\delta_{b}^{j_{k}} U_{i_{1} i_{2} \ldots i_{l}}^{j_{1} j_{2} \ldots a} .
\end{aligned}
$$

The collection $\left\{\Lambda_{b}^{a}(Y)\right\}_{a, b=1}^{n}$ is an orthornormal basis for each $\mathcal{V}_{Y}$ with respect to (the restriction of) $g^{F}$, but these vector fields will not in general be parallel to $\mathcal{O}(M)$. Thus it is sometimes convenient to consider instead the vectors

$$
\rho_{a b} \doteqdot \delta_{a c} \Lambda_{b}^{c}-\delta_{b c} \Lambda_{a}^{c} .
$$

It is easily checked that the set $\left\{\rho_{a b}\right\}_{a<b}$ is an orthogonal basis for $T_{Y} \mathcal{O}(M)_{\pi(x)}$.

Next we define a global frame spanning the horizontal subbundle $\mathcal{W} \subset T F(M)$. Given any $x \in M$, vector field $X \in T_{x} M$, and frame $Y=\left(Y_{1}, Y_{2}, \ldots, Y_{n}\right) \in F(M)_{x}$, define

$$
\gamma_{X}(t) \doteqdot\left(\tau_{\sigma(t)} Y_{1}, \tau_{\sigma(t)} Y_{2}, \ldots, \tau_{\sigma(t)} Y_{n}\right)
$$

where $\sigma(t)$ is any path in $M$ with $\sigma(0)=x$ and $\dot{\sigma}(t)=X$ and $\tau_{\sigma(t)}: T_{x} M \rightarrow T_{\sigma(t)} M$ is parallel transport along $\sigma(t)$. Then we define, for $Y \in F(M)$, and any $a=1,2, \ldots, n$,

$$
\left.\left.\nabla_{a}\right|_{Y} \doteqdot \frac{d}{d t}\right|_{t=0} \gamma_{Y_{a}}(t)
$$

That is, we define $\left.\nabla_{a}\right|_{Y}$ to be the horizontal lift of $Y_{a}$ at $Y \in F(M)_{x}$.

Local coordinates $\left\{x^{i}\right\}_{i=1}^{n}$ on $M$ define local coordinates $\left(\tilde{x}^{i}, y_{a}^{i}\right)$ on $F(M)$ by $\tilde{x}^{i}=x^{i} \circ \pi$ and

In these coordinates,

$$
Y_{a}=y_{a}^{i}(Y) \frac{\partial}{\partial x^{i}}
$$

$$
\nabla_{a}=y_{a}^{j}(Y)\left(\frac{\partial}{\partial \tilde{x}^{j}}-y_{b}^{k} \Gamma_{k j}^{i}(\pi(Y)) \frac{\partial}{\partial y_{b}^{i}}\right) .
$$

Thus, for example, on a two-tensor $U$,

$$
\nabla_{k}\left(U_{i j}\right)=(\nabla U)_{k i j}=\nabla_{k} U_{i j}
$$


where the leftmost expression represents the action of $\nabla_{k} \in T F(M)$ on the $\mathbb{R}^{n^{2}}$ valued function on $F(M)$, the middle expression represents the value of the $\mathbb{R}^{n^{3}}$ valued function $\nabla U$ on $F(M)$, and the rightmost expression represents the tensor $\nabla U(x)$ evaluated at $Y_{k}(x), Y_{i}(x)$, and $Y_{j}(x)$. Where the interpretation is clear from the context, we will the notation of the rightmost expression to represent all three cases. The set $\left\{\left.\nabla_{a}\right|_{Y}\right\}_{a=1}^{n}$ is a basis for the horizontal space $\mathcal{W}_{Y} \subset T_{Y} F(M)$ at each $Y$. Since $\nabla_{a} g_{i j}=0$, they are also tangent to $T_{Y} \mathcal{O}(M)$.

Finally we consider differentiation in the time direction. As the vector $\frac{\partial}{\partial t} \in$ $T \widetilde{F(M)}$ is not in general tangent to $\widetilde{\mathcal{O}(M)}$, it is convenient to work instead with the vector

$$
D_{t} \doteqdot \frac{\partial}{\partial t}+R_{a b} g^{b c} \Lambda_{c}^{a}
$$

which satisfies $D_{t} g_{i j}=0$. On $\widetilde{\mathcal{O}(M)}$, it is given simply by

$$
D_{t}=\frac{\partial}{\partial t}+R_{a c} \Lambda_{c}^{a}
$$

As remarked in Section 3, extending a tensor field $V$ defined on some time-slice to a time-dependent family via the $\operatorname{ODE}(3.1)$ is equivalent to solving $D_{t} V \equiv 0$. In particular, for the projections $\bar{P}$ and $\widehat{P}$, we have

$$
D_{t} \bar{P}_{a b c d} \equiv D_{t} \widehat{P}_{a b c d} \equiv 0 \text {. }
$$

The collection $\left\{D_{t}\right\} \cup\left\{\nabla_{a}\right\}_{a=1}^{n} \cup\left\{\rho_{a b}\right\}_{1 \leq a<b \leq n}$, forms the global frame field for $\widetilde{T \mathcal{O}(M)}$ with respect to which we will perform our calculations (although it will be convenient to use of all elements of the set $\left\{\rho_{a b}\right\}_{1 \leq a, b \leq n}$, i.e., including $\rho_{a b}$ for $a \geq b$ ). As derivations on the frame bundle, they satisfy the following commutator relations.

Lemma 4.3. Restricted to $\widetilde{\mathcal{O}(M)}$, the vectors $D_{t}, \Lambda_{b}^{a}, \rho_{a b}$ and $\nabla_{a}$ satisfy

$$
\begin{aligned}
{\left[\Lambda_{b}^{a}, \nabla_{c}\right] } & =\delta_{c}^{b} \nabla_{a}, \\
{\left[\rho_{a b}, \nabla_{c}\right] } & =\delta_{a c} \nabla_{b}-\delta_{b c} \nabla_{a}, \\
{\left[D_{t}, \nabla_{a}\right] } & =\nabla_{b} R_{a c} \rho_{b c}+R_{a c} \nabla_{c}=\nabla_{p} R_{p a c b} \Lambda_{c}^{b}+R_{a c} \nabla_{c}, \\
{\left[D_{t}-\Delta, \nabla_{a}\right] } & =R_{a b d c} \nabla_{b} \rho_{c d}=2 R_{a b d c} \Lambda_{d}^{c} \nabla_{b}+2 R_{a b} \nabla_{b} .
\end{aligned}
$$

Here $\Delta=\nabla_{p} \nabla_{p}=\sum_{p=1}^{n} \nabla_{p} \nabla_{p}$.

Proof. Equations (4.8), (4.9), and the first equalities in (4.10) and (4.11) appear in Appendix F of [CCG2]. For the second equality in (4.10), we compute

$$
\begin{aligned}
\nabla_{b} R_{a c} \rho_{b c} & =\nabla_{b} R_{a c}\left(\delta_{b p} \Lambda_{c}^{p}-\delta_{c p} \Lambda_{b}^{p}\right) \\
& =\left(\nabla_{p} R_{a c}-\nabla_{c} R_{a p}\right) \Lambda_{c}^{p} \\
& =\nabla_{p} R_{p a c b} \Lambda_{c}^{b} .
\end{aligned}
$$

For the second equality in (4.11), we compute

$$
\begin{aligned}
R_{a b d c} \nabla_{b} \rho_{c d} & =R_{a b d c}\left(\rho_{c d} \nabla_{b}+\left[\nabla_{b}, \rho_{c d}\right]\right) \\
& =R_{a b d c}\left(\left(\delta_{c e} \Lambda_{d}^{e}-\delta_{d e} \Lambda_{c}^{e}\right) \nabla_{b}+\left(\delta_{d b} \nabla_{c}-\delta_{c b} \nabla_{d}\right)\right) \\
& =2\left(R_{a b d c} \Lambda_{c}^{d}+R_{a b}\right) \nabla_{b},
\end{aligned}
$$

using (4.9) in the second line.

q.e.d. 
4.4. Evolution equations for $A$ and $B$. We begin by computing the evolution equations for the components of the ordinary-differential component of our system. We will need the following consequence of Lemma 3.2.

Lemma 4.4. The projections $\bar{P}$ and $\widehat{P}$ satisfy

$$
\bar{P}_{a b c d} \Lambda_{c}^{d} \widehat{P}_{i j k l}=0 .
$$

Proof. Note that

$$
\begin{aligned}
\bar{P}_{a b c d} \Lambda_{c}^{d} \widehat{P}_{i j k l}= & \bar{P}_{a b d i} \widehat{P}_{d j k l}+\bar{P}_{a b d j} \widehat{P}_{i d k l}+\bar{P}_{a b d k} \widehat{P}_{i j d l}+\bar{P}_{a b d l} \widehat{P}_{i j k d} \\
= & -\bar{P}_{a b i d} \widehat{P}_{k l d j}+\bar{P}_{a b d j} \widehat{P}_{k l i d}-\bar{P}_{a b k d} \widehat{P}_{i j d l}+\bar{P}_{a b d l} \widehat{P}_{i j k d} \\
= & \left\langle\left[\widehat{P}\left(e_{k} \wedge e_{l}\right), \bar{P}\left(e_{a} \wedge e_{b}\right)\right], e_{i} \wedge e_{j}\right\rangle \\
& \quad+\left\langle\left[\widehat{P}\left(e_{i} \wedge e_{j}\right), \bar{P}\left(e_{a} \wedge e_{b}\right)\right], e_{k} \wedge e_{l}\right\rangle .
\end{aligned}
$$

In view of Lemma 3.2, we have $[K(t), H(t)] \subset K(t)$, thus

$$
\begin{aligned}
\bar{P}_{a b c d} \Lambda_{c}^{d} \widehat{P}_{i j k l}=\langle & {\left.\left[\widehat{P}\left(e_{k} \wedge e_{l}\right), \bar{P}\left(e_{a} \wedge e_{b}\right)\right], \widehat{P}\left(e_{i} \wedge e_{j}\right)\right\rangle } \\
& +\left\langle\left[\widehat{P}\left(e_{i} \wedge e_{j}\right), \bar{P}\left(e_{a} \wedge e_{b}\right)\right], \widehat{P}\left(e_{k} \wedge e_{l}\right)\right\rangle
\end{aligned}
$$

which vanishes on account of antisymmetry of the map $(X, Y, Z) \mapsto\langle[X, Y], Z\rangle$. q.e.d.

In view of (4.7), the only non-zero contributions to the evolution equations for $A=\nabla \widehat{P}$ and $B=\nabla \nabla \widehat{P}$ come from the time-dependency of the connection. These contributions are encoded in the commutators of $D_{t}$ with the horizontal vectors $\nabla_{a}$.

Proposition 4.5. Regarded as a matrix-valued function on $\widetilde{\mathcal{O}(M)}$, the tensor $A$ evolves according to

$$
\begin{aligned}
D_{t} A_{m i j k l}= & R_{m r} A_{r i j k l}-\widehat{P}_{p j k l} \widehat{T}_{r p i r m}-\widehat{P}_{i p k l} \widehat{T}_{r p j r m} \\
& -\widehat{P}_{i j p l} \widehat{T}_{r p k r m}-\widehat{P}_{i j k p} \widehat{T}_{r p l r m} .
\end{aligned}
$$

Proof. Since $D_{t} \widehat{P}_{i j k l}=0$, we have $D_{t} A_{m i j k l}=D_{t} \nabla_{m} \widehat{P}_{i j k l}=\left[D_{t}, \nabla_{m}\right] \widehat{P}_{i j k l}$. Thus from (4.10) we have

$$
D_{t} A_{m i j k l}=R_{m r} A_{r i j k l}+T_{r r m p q} \Lambda_{p}^{q} \widehat{P}_{i j k l} .
$$

Now,

$$
\begin{aligned}
T_{\text {rrmpq }} & =T_{\text {rrmuv }}\left(\widehat{P}_{u v p q}+\bar{P}_{u v p q}\right) \\
& =-\widehat{T}_{r p q r m}+T_{\text {rrmuv }} \bar{P}_{u v p q},
\end{aligned}
$$

so

$$
\begin{aligned}
T_{r r m p q} \Lambda_{p}^{q} \widehat{P}_{i j k l} & =-\widehat{T}_{r p q r m} \Lambda_{p}^{q} \widehat{P}_{i j k l}+T_{r r m u v} \bar{P}_{u v p q} \Lambda_{p}^{q} \widehat{P}_{i j k l} \\
& =-\widehat{T}_{r p q r m} \Lambda_{p}^{q} \widehat{P}_{i j k l},
\end{aligned}
$$

on account of Lemma 4.4, and (4.13) follows.

q.e.d. 
Proposition 4.6. Regarded as a matrix-valued function on $\widetilde{\mathcal{O}(M)}$, the tensor $B$ evolves according to

$$
\begin{aligned}
& D_{t} B_{m n i j k l}=R_{m r} B_{r n i j k l}+R_{n r} B_{m r i j k l}+\nabla_{n} R_{m s} A_{s i j k l} \\
& \quad+T_{r r m s i} A_{n s j k l}+T_{r r m s j} A_{n i s k l}+T_{r r m s k} A_{n i j s l}+T_{r r m s l} A_{n i j k s} \\
& \quad-\widehat{P}_{s j k l} \nabla_{m} \widehat{T}_{r s i r n}-\widehat{P}_{i s k l} \nabla_{m} \widehat{T}_{r s j r n}-\widehat{P}_{i j s l} \nabla_{m} \widehat{T}_{r s k r n}-\widehat{P}_{i j k s} \nabla_{m} \widehat{T}_{r s l r n} \\
& \quad+T_{r r n v w}\left(\widehat{P}_{s j k l} A_{m v w s i}+\widehat{P}_{i s k l} A_{m v w s j}+\widehat{P}_{i j s l} A_{m v w s k}+\widehat{P}_{i j k s} A_{m v w s l}\right) .
\end{aligned}
$$

Proof. As before, $D_{t} B_{m n i j k l}=\left[D_{t}, \nabla_{m} \nabla_{n}\right] \widehat{P}_{i j k l}$. We compute this commutator using a double application of (4.10):

$$
\begin{aligned}
{\left[D_{t}, \nabla_{m} \nabla_{n}\right]=} & {\left[D_{t}, \nabla_{m}\right] \nabla_{n}+\nabla_{m}\left[D_{t}, \nabla_{n}\right] } \\
= & \left(R_{m r} \nabla_{r}+T_{r r m s u} \Lambda_{s}^{u}\right) \nabla_{n}+\nabla_{m}\left(R_{n r} \nabla_{r}+T_{r r n s u} \Lambda_{s}^{u}\right) \\
= & R_{m r} \nabla_{r} \nabla_{n}+T_{r r m s u} \Lambda_{s}^{u} \nabla_{n}+\nabla_{m} R_{n r} \nabla_{r}+R_{n r} \nabla_{m} \nabla_{r} \\
& +\nabla_{m} T_{r r n s u} \Lambda_{s}^{u}+T_{r r n s u} \nabla_{m} \Lambda_{s}^{u} .
\end{aligned}
$$

Then, using (4.8), we have $T_{r r n s u} \nabla_{m} \Lambda_{s}^{u}=-T_{r r n s m} \nabla_{s}=T_{r r n m s} \nabla_{s}$, so

$$
\begin{aligned}
D_{t} B_{m n i j k l}= & R_{m r} B_{r n i j k l}+R_{n r} B_{m r i j k l}+\nabla_{m} T_{r r n s u} \Lambda_{s}^{u} \widehat{P}_{i j k l} \\
& +\left(\nabla_{m} R_{n s}+T_{r r n m s}\right) A_{s i j k l}+T_{r r m s u} \Lambda_{s}^{u} A_{n i j k l} .
\end{aligned}
$$

Now, since $\bar{P}+\widehat{P}=\mathrm{Id}$, we have $A=\nabla \widehat{P}=-\nabla \bar{P}$; applying this and considering the decomposition of $T$ into components as above, we compute

$$
\begin{aligned}
\nabla_{m} T_{\text {rrnsu }} & =\nabla_{m}\left(-\widehat{T}_{\text {rsurn }}+T_{\text {rrnvw }} \bar{P}_{v w s u}\right) \\
& =-\nabla_{m} \widehat{T}_{\text {rsurn }}-T_{\text {rrnvw }} A_{\text {mvwsu }}+\nabla_{m} T_{\text {rrnvw }} \bar{P}_{v w s u} .
\end{aligned}
$$

Using Lemma 4.4 again, we therefore have

$$
\nabla_{m} T_{r r n s u} \Lambda_{s}^{u} \widehat{P}_{i j k l}=-\left(\nabla_{m} \widehat{T}_{r s u r n}+T_{r r n v w} A_{m v w s u}\right) \Lambda_{s}^{u} \widehat{P}_{i j k l} .
$$

Finally, we can simplify the last line of (4.15). The last term is

$$
\begin{aligned}
T_{r r m s u} \Lambda_{s}^{u} A_{n i j k l}= & T_{r r m s n} A_{s i j k l}+T_{r r m s i} A_{n s j k l}+T_{r r m s j} A_{n i s k l} \\
& +T_{r r m s k} A_{n i j s l}+T_{r r m s l} A_{n i j k s},
\end{aligned}
$$

and

$$
\begin{aligned}
\nabla_{m} R_{n s}+T_{r r n m s}+T_{r r m s n} & =\nabla_{m} R_{n s}+\left(\nabla_{s} R_{m n}-\nabla_{m} R_{s n}\right)+\left(\nabla_{n} R_{s m}-\nabla_{s} R_{n m}\right) \\
& =\nabla_{n} R_{m s}
\end{aligned}
$$

so the last line reduces to

$$
\begin{aligned}
& \left(\nabla_{m} R_{n s}+T_{r r n m s}\right) A_{s i j k l}+T_{r r m s u} \Lambda_{s}^{u} A_{n i j k l} \\
& =\nabla_{n} R_{m s} A_{s i j k l}+T_{r r m s i} A_{n s j k l}+T_{r r m s j} A_{n i s k l} \\
& \quad+T_{r r m s k} A_{n i j s l}+T_{r r m s l} A_{n i j k s} .
\end{aligned}
$$

Combining (4.15), (4.16), and(4.17), we then obtain (4.14).

q.e.d.

4.5. Evolution equations for $R$ and $T$. Recall that for $A, B \in \operatorname{End}\left(\wedge^{2} T_{p}^{*} M\right)$, one can form the product $A \# B \in \operatorname{End}\left(\wedge^{2} T_{p}^{*} M\right) \cong \wedge^{2} T_{p}^{*} M^{*} \otimes \wedge^{2} T_{p}^{*} M^{*}$ defined by

$$
A \# B(\omega) \doteqdot \frac{1}{2} \sum_{M, N}\left\langle\left[A\left(\varphi^{M}\right), B\left(\varphi^{N}\right)\right], \omega\right\rangle \cdot\left[\varphi^{M}, \varphi^{N}\right]
$$

where $\left\{\varphi_{\alpha}\right\}$ is an orthonormal basis for $\wedge^{2} T_{p}^{*} M$. This product is bilinear and symmetric in its arguments, and with it, we define the square $A^{\#} \doteqdot A \# A$. In terms 
of the structure constants $\left[\varphi^{M}, \varphi^{N}\right]=C_{P}^{M N} \varphi^{P}$ (and regarded as an element of $\left.\wedge^{2} T_{p}^{*} M^{*} \otimes \wedge^{2} T_{p}^{*} M^{*}\right)$, we have $(A \# B)_{I J}=(1 / 2) A_{M P} B_{N Q} C_{I}^{P Q} C_{J}^{M N}$.

Now define

$$
\mathcal{Q}: \operatorname{End}\left(\wedge^{2} T^{*} M\right) \rightarrow \operatorname{End}\left(\wedge^{2} T^{*} M\right)
$$

by

$$
\mathcal{Q}(A) \doteqdot A^{2}+A^{\#}
$$

and

$$
\mathcal{S}: \operatorname{End}\left(\wedge^{2} T^{*} M\right) \times\left(T M^{*} \otimes \operatorname{End}\left(\wedge^{2} T^{*} M\right)\right) \rightarrow T M^{*} \otimes \operatorname{End}\left(\wedge^{2} T^{*} M\right)
$$

by

$$
\mathcal{S}(A, F)(X, \cdot) \doteqdot A \circ(F\lrcorner X)+(F\lrcorner X) \circ A+2(F\lrcorner X) \# A .
$$

These operators arise as reaction terms in the evolution equations for $R$ and $T$.

Proposition 4.7. Viewed as matrix-valued functions on $\widetilde{\mathcal{O}(M)}$, the tensors $R$ and $T$ evolve according to

$$
\left(D_{t}-\Delta\right) R_{i j k l}=-\mathcal{Q}(\mathrm{Rm})_{i j k l},
$$

$$
\left(D_{t}-\Delta\right) T_{m i j k l}=2 R_{m b} T_{b i j k l}+2 R_{m b d p} \Lambda_{d}^{p} T_{b i j k l}-\mathcal{S}(\mathrm{Rm}, \nabla \mathrm{Rm})_{m i j k l} .
$$

Proof. Equation (4.20) is standard. For (4.21), we use (4.20) and (4.11):

$$
\begin{aligned}
\left(D_{t}-\Delta\right) T_{m i j k l} & =\left[D_{t}-\Delta, \nabla_{m}\right] R_{i j k l}+\nabla_{m}\left(D_{t}-\Delta\right) R_{i j k l} \\
& =2\left(R_{m b} \nabla_{b}+R_{m b d p} \Lambda_{d}^{p} \nabla_{b}\right) R_{i j k l}-\nabla_{m}(\mathcal{Q}(\mathrm{Rm})) \\
& =2 R_{m b} T_{b i j k l}+2 R_{m b d p} \Lambda_{d}^{p} T_{b i j k l}-\nabla_{m}(\mathcal{Q}(\mathrm{Rm})) .
\end{aligned}
$$

For the last term, note that at any $p \in M$,

$$
\begin{aligned}
\nabla(\mathcal{Q}(\mathrm{Rm}))\left(e_{m}, \cdot\right) & =\nabla_{m} \mathrm{Rm} \circ \mathrm{Rm}+\mathrm{Rm} \circ \nabla_{m} \mathrm{Rm}+\mathrm{Rm} \# \nabla_{m} \mathrm{Rm} \\
& =\mathcal{S}(\mathrm{Rm}, \nabla \mathrm{Rm})\left(e_{m}, \cdot\right)
\end{aligned}
$$

in view of the symmetry of the product \#.

q.e.d.

REMARK 4.8. We choose to leave the terms $2 R_{m b} T_{b i j k l}+2 R_{m b d p} \Lambda_{d}^{p} T_{b i j k l}$ in (4.21) in a rather raw form for convenience in a later computation, however, we might alternatively have written

$$
\begin{aligned}
R_{m b} T_{b i j k l}+R_{m b d p} \Lambda_{d}^{p} T_{b i j k l} & =R_{m b d i} T_{b d j k l}+R_{m b d j} T_{b i d k l}+R_{m b d k} T_{b i j d l}+R_{m b d l} T_{b i j k d} \\
& \doteqdot \mathcal{U}(\mathrm{Rm}, \nabla \mathrm{Rm})_{m i j k l}
\end{aligned}
$$

where

$$
\mathcal{U}: \operatorname{End}\left(\wedge^{2} T^{*} M\right) \times\left(T M^{*} \otimes \operatorname{End}\left(\wedge^{2} T^{*} M\right)\right) \rightarrow T M^{*} \otimes \wedge^{2} T^{*} M \otimes \wedge^{2} T^{*} M
$$

is given by

$$
\mathcal{U}(A, F)(X, \omega, \eta)=\sum_{i=1}^{n}\left(\left\langle\left[A\left(e_{i} \wedge X\right), F\left(e_{i}, \omega\right)\right], \eta\right\rangle+\left\langle\left[A\left(e_{i} \wedge X\right), F\left(e_{i}, \eta\right)\right], \omega\right\rangle\right)
$$

in the fiber over $p$ for $\left\{e_{i}\right\}$ an orthonormal basis of $T_{p} M$. Alternatively, using the second Bianchi identity and the symmetries of $T$, one can define the tensor $C_{m i j k l} \doteqdot$ $-T_{m i p q j} R_{k p q l}$ (analogous to Hamilton's $B_{i j k l}=-R_{i p q j} R_{k p q l}$ ) and write the evolution of $T$ in the form

$$
\begin{aligned}
& \left(D_{t}-\Delta\right) T_{m i j k l} \\
& \quad=2\left(C_{m i j k l}+C_{m k l i j}-C_{m i j l k}-C_{m l k i j}+C_{m i k j l}+C_{m j l i k}-C_{m i l j k}-C_{m j k i l}\right) \\
& \quad+2\left(C_{k l j m i}-C_{l k j m i}+C_{l k i m j}-C_{k l i m j}+C_{i j l m k}-C_{j i l m k}+C_{j i k m l}-C_{i j k m l}\right) .
\end{aligned}
$$


If $\mathcal{P}$ denotes the projection $T_{4}(M) \rightarrow \wedge^{2} T^{*} M \otimes_{S} \wedge^{2} T^{*} M$ (where $\otimes_{S}$ denotes the symmetric tensor product), that is,

$$
\mathcal{P}(V)_{i j k l}=\frac{1}{8}\left(V_{i j k l}-V_{j i k l}-V_{i j l k}+V_{j i k l}+V_{k l i j}-V_{l k i j}-V_{k l j i}+V_{l k j i}\right),
$$

then the sum in parantheses on the last line in the expression above (which corresponds to $\mathcal{U}(\mathrm{Rm}, \nabla \mathrm{Rm}))$ is $\left.-8 \cdot \mathcal{P}(C\lrcorner_{4} e_{m}\right)$ where $\lrcorner_{i} X$ denotes inner multiplication by $X$ in the $i$-th argument.

4.6. Evolution equations for $\widehat{R}$ and $\widehat{T}$. Using the results of the preceding section, we now compute the evolutions of the components of the parabolic portion of our PDE-ODE system. We begin with the consideration of the reaction terms $\mathcal{Q}(\mathrm{Rm})$ and $\mathcal{S}(\mathrm{Rm}, \nabla \mathrm{Rm})$.

Lemma 4.9. Denote temporarily $R=\mathrm{Rm}, T=\nabla \mathrm{Rm}$. At any $p \in M$,

$$
\mathcal{Q}(R) \circ \widehat{P}=R \circ \widehat{R}+\bar{R}^{*} \# \widehat{R}^{*}+\mathrm{Rm} \# \widehat{R}^{*}
$$

$$
(\mathcal{S}(R, T)\lrcorner X) \circ \widehat{P}=R \circ \widehat{T}_{X}+T_{X} \circ \widehat{R}+\left(\widehat{T}_{X}^{*} \# \bar{R}^{*}+T_{X} \# \widehat{R}^{*}\right) \circ \widehat{P}
$$

for any $X \in T_{p} M$, where we use the shorthand

$$
\left.\left.T_{X} \doteqdot T\right\lrcorner X \quad \widehat{T}_{X} \doteqdot \widehat{T}\right\lrcorner X \in \operatorname{End}\left(\wedge^{2} T_{p}^{*} M\right)
$$

and denote the adjoint of an operator $A \in \operatorname{End}\left(\wedge^{2} T_{p}^{*} M\right)$ by $A^{*}$.

REMARK 4.10. As we observed in (4.1) and (4.2), the operators $\bar{R}, \widehat{R}, \bar{T}, \widehat{T}$ are no longer self-adjoint. However, $\bar{R}^{*}=\bar{P} \circ R, \widehat{R}^{*}=\widehat{P} \circ R, \bar{T}_{X}^{*}=\bar{P} \circ T_{X}, \widehat{T}_{X}^{*}=\widehat{P} \circ T_{X}$. In coordinates, for example, $\bar{R}_{i j k l}^{*}=\bar{P}_{a b k l} R_{i j b a}$, and similarly for the others.

Proof. The origin of the first term in (4.22) is clear. For the second, we use $\bar{P}+\widehat{P}=\mathrm{Id}$ and expand to find

$$
\begin{aligned}
R \# R & =((\widehat{P}+\bar{P}) \circ R) \#((\widehat{P}+\bar{P}) \circ R) \\
& =(\bar{P} \circ R) \#(\bar{P} \circ R)+(\widehat{P} \circ R) \#(\bar{P} \circ R)+(\bar{P} \circ R) \#(\widehat{P} \circ R)+(\widehat{P} \circ R) \#(\widehat{P} \circ R) \\
& =\bar{R}^{*} \# \bar{R}^{*}+2 \bar{R}^{*} \# \widehat{R}^{*}+\widehat{R}^{*} \# \widehat{R}^{*} .
\end{aligned}
$$

We claim $\left(\bar{R}^{*} \# \bar{R}^{*}\right) \circ \widehat{P} \equiv 0$. To see this, let $p \in M$ and $\left\{\varphi^{A}\right\}$ be an orthogonal basis for $\wedge^{2}\left(T_{p} M\right)$. Then for any $1 \leq N \leq n(n-1) / 2$, we have

$$
\begin{aligned}
\left(\bar{R}^{*} \# \bar{R}^{*}\right)\left(\widehat{P}\left(\varphi^{N}\right)\right) & =\frac{1}{2} \sum_{A, B}\left\langle\left[\bar{P}\left(R\left(\varphi^{A}\right)\right), \bar{P}\left(R\left(\varphi^{B}\right)\right)\right], \widehat{P}\left(\varphi^{N}\right)\right\rangle \cdot\left[\varphi^{A}, \varphi^{B}\right] \\
& =\frac{1}{2} \sum_{A, B} \mathcal{T}[\bar{P}, \bar{P}, \widehat{P}]\left(R\left(\varphi^{A}\right), R\left(\varphi^{B}\right), \varphi^{N}\right) \cdot\left[\varphi^{A}, \varphi^{B}\right]
\end{aligned}
$$

which vanishes by Lemma 3.4. Thus

$$
\begin{aligned}
\left(R^{2}+R^{\#}\right) \circ \widehat{P} & =R \circ \widehat{R}+\left(2 \bar{R}^{*} \# \widehat{R}^{*}+\widehat{R}^{*} \# \widehat{R}^{*}\right) \circ \widehat{P} \\
& =R \circ \widehat{R}+\left(\bar{R}^{*} \# \widehat{R}^{*}+R \# \widehat{R}^{*}\right) \circ \widehat{P}
\end{aligned}
$$

We argue similarly for (4.23). Again the first two terms are clear, and for the term involving the Lie-algebraic product, we expand into components relative to the decomposition $\wedge^{2} T_{p}^{*} M=H_{p} \oplus K_{p}$ :

$$
\begin{aligned}
T_{X} \# R & =\left(\bar{T}_{X}^{*}+\widehat{T}_{X}^{*}\right) \#\left(\bar{R}^{*}+\widehat{R}^{*}\right) \\
& =\bar{T}_{X}^{*} \# \bar{R}^{*}+\widehat{T}_{X}^{*} \# \bar{R}^{*}+T_{X} \# \widehat{R}^{*} .
\end{aligned}
$$


Then, just as before,

$$
\left(\bar{T}_{X}^{*} \# \bar{R}^{*}\right) \circ \widehat{P}\left(\varphi^{N}\right)=\frac{1}{2} \sum_{A, B} \mathcal{T}[\bar{P}, \bar{P}, \widehat{P}]\left(T\left(X, \varphi^{A}\right), R\left(\varphi^{B}\right), \varphi^{N}\right) \cdot\left[\varphi^{A}, \varphi^{B}\right],
$$

and so is zero for all $N$ by Lemma 3.4.

q.e.d.

REMARK 4.11. As functions on the frame bundle, we have

$$
\begin{aligned}
(\mathcal{Q}(\mathrm{Rm}) \circ \widehat{P})_{i j k l}= & R_{c d l k} \widehat{R}_{i j c d}+2 \widehat{P}_{i j c e}\left(\bar{R}_{c p q l}^{*} \widehat{R}_{e p q k}-\bar{R}_{c p q k}^{*} \widehat{R}_{e p q l}\right) \\
& +2 \widehat{P}_{i j c e}\left(R_{c p k q} \widehat{R}_{e p q l}^{*}-R_{c p l q} \widehat{R}_{e p q k}^{*}\right)
\end{aligned}
$$

and

$$
\begin{aligned}
(\mathcal{S}(\mathrm{Rm}, \nabla T) \circ(\operatorname{Id} \times \widehat{P}))_{m i j k l}= & R_{a b l k} \widehat{T}_{m i j a b}+T_{m a b l k} \widehat{R}_{i j a b} \\
& +2 \widehat{P}_{i j c e}\left(\bar{R}_{c p q l}^{*} \widehat{T}_{m e p q k}^{*}-\bar{R}_{c p q k}^{*} \widehat{T}_{m e p q l}\right) \\
& +2 \widehat{P}_{i j c e}\left(T_{m c p k q} \widehat{R}_{e p q l}^{*}-T_{m c p l q} \widehat{R}_{e p q k}^{*}\right) .
\end{aligned}
$$

Proposition 4.12. The tensor $\widehat{R}$, regarded as a matrix-valued function on $\widetilde{\mathcal{O}(M)}$, evolves according to

$$
\left(D_{t}-\Delta\right) \widehat{R}_{i j k l}=2 A_{p i j a b} T_{p a b k l}+B_{p p i j a b} R_{a b k l}+(\mathcal{Q}(\mathrm{Rm}) \circ \widehat{P})_{i j k l} .
$$

Proof. We have

$$
\begin{aligned}
\left(D_{t}-\Delta\right) \widehat{R} & =\operatorname{Rm} \circ\left(D_{t}-\Delta\right) \widehat{P}-2 \nabla_{p} \operatorname{Rm} \circ \nabla_{p} \widehat{P}+\left(D_{t}-\Delta\right) \operatorname{Rm} \circ \widehat{P} \\
& =-\operatorname{Rm} \circ \Delta \widehat{P}-2 \nabla_{p} \operatorname{Rm} \circ \nabla_{p} \widehat{P}+\mathcal{Q}(\mathrm{Rm}) \circ \widehat{P}
\end{aligned}
$$

using (4.7) and (4.20). Then

$$
-(\operatorname{Rm} \circ \Delta \widehat{P})_{i j k l}=-\Delta \widehat{P}_{i j a b} R_{a b l k}=B_{p p i j a b} R_{a b k l},
$$

and

$$
-2\left(\nabla_{p} \operatorname{Rm} \circ \nabla_{p} \widehat{P}\right)_{i j k l}=-2 \nabla_{p} \widehat{P}_{i j a b} \nabla_{p} R_{a b l k}=2 A_{p i j a b} T_{p a b k l}
$$

and (4.26) follows.

q.e.d.

Proposition 4.13. The tensor $\widehat{T}$, viewed as a matrix-valued function on $\widetilde{\mathcal{O}(M)}$, evolves according to

$$
\begin{aligned}
\left(D_{t}\right. & -\Delta) \widehat{T}_{m i j k l} \\
= & 2 A_{p i j a b} \nabla_{p} T_{m i j k l}+B_{p p i j a b} T_{m a b k l}+(\mathcal{S}(R, T) \circ(\operatorname{Id} \times \widehat{P}))_{m i j k l} \\
& +2\left(R_{m p q i} \widehat{T}_{p q j k l}+R_{m p q j} \widehat{T}_{p i q k l}+R_{m p q k} \widehat{T}_{p i j q l}+R_{m p q l} \widehat{T}_{p i j k q}\right) \\
& +2\left(\widehat{R}_{q i m p} \widehat{T}_{p q j k l}+\widehat{R}_{q j m p} \widehat{T}_{p i q k l}+T_{p a b l k}\left(\widehat{P}_{i j q b} \widehat{R}_{q a m p}+\widehat{P}_{i j a q} \widehat{R}_{q b m p}\right)\right) .
\end{aligned}
$$

Proof. We obtain the evolution equation for $\widehat{T}$ by a computation similar to that for $\widehat{R}$. Namely, we have $\widehat{T}_{m i j k l}=-\widehat{P}_{i j a b} T_{m a b k l}$, and, as before,

(4.28) $\left(D_{t}-\Delta\right) \widehat{T}_{m i j k l}=\widehat{P}_{i j a b}\left(D_{t}-\Delta\right) T_{m a b l k}+2 A_{p i j a b} \nabla_{p} T_{m a b k l}+B_{\text {ppijab }} T_{m a b k l}$.

By (4.21), we have

$$
\left(D_{t}-\Delta\right) T_{m a b l k}=2\left(R_{m p}+R_{m p q r} \Lambda_{q}^{r}\right) T_{p a b l k}-\mathcal{S}(\mathrm{Rm}, \nabla \mathrm{Rm})_{m a b l k},
$$

and

$$
\mathcal{S}(\mathrm{Rm}, \nabla \mathrm{Rm})_{m a b l k} \widehat{P}_{i j a b}=-(\mathcal{S}(R, T) \circ(I d \times \widehat{P}))_{m i j k l},
$$

so we just need to consider the contraction of first term in (4.29) against $\widehat{P}_{i j a b}$. 
First, since $\Lambda_{q}^{r}$ is a derivation, we can write

$$
\widehat{P}_{i j a b} \Lambda_{q}^{r} T_{m a b l k}=\Lambda_{q}^{r} \widehat{T}_{m i j k l}-T_{m a b l k} \Lambda_{q}^{r} \widehat{P}_{i j a b} .
$$

Also,

$$
R_{m p q r}=R_{q r m p}=\left(\widehat{P}_{q r u v}+\bar{P}_{q r u v}\right) R_{u v m p}=\widehat{R}_{q r p m}+\bar{P}_{u v q r} R_{u v m p}
$$

so

$$
\begin{aligned}
\widehat{P}_{i j a b} R_{m p q r} \Lambda_{q}^{r} T_{m a b l k} & =R_{m p q r} \Lambda_{q}^{r} \widehat{T}_{m i j k l}-T_{m a b l k}\left(\widehat{R}_{q r p m}+\bar{P}_{u v q r} R_{u v m p}\right) \Lambda_{q}^{r} \widehat{P}_{i j a b} \\
& =R_{m p q r} \Lambda_{q}^{r} \widehat{T}_{m i j k l}-T_{m a b l k} \widehat{R}_{q r p m} \Lambda_{q}^{r} \widehat{P}_{i j a b}
\end{aligned}
$$

by Lemma 4.4. With this, we can expand to obtain

$$
\begin{aligned}
\widehat{P}_{i j a b} & \left(R_{m p}+R_{m p q r} \Lambda_{q}^{r}\right) T_{p a b l k} \\
= & R_{m p q i} \widehat{T}_{m q j k l}+R_{m p q j} \widehat{T}_{m i q k l}+R_{m p q k} \widehat{T}_{m i j k q l}+R_{m p q l} \widehat{T}_{m i j k q} \\
& -T_{m a b l k}\left(\widehat{P}_{q j a b} \widehat{R}_{q i p m}+\widehat{P}_{i q a b} \widehat{R}_{q j p m}+\widehat{P}_{i j q b} \widehat{R}_{q a p m}+\widehat{P}_{i j a q} \widehat{R}_{q b p m}\right) \\
= & R_{m p q i} \widehat{T}_{m q j k l}+R_{m p q j} \widehat{T}_{m i q k l}+R_{m p q k} \widehat{T}_{m i j k q l}+R_{m p q l} \widehat{T}_{m i j k q} \\
& -\widehat{T}_{m q j k l} \widehat{R}_{q i p m}-\widehat{T}_{m i q k l} \widehat{R}_{q j p m}+T_{m a b k l}\left(\widehat{P}_{i j q b} \widehat{R}_{q a p m}+\widehat{P}_{i j a q} \widehat{R}_{q b p m}\right) .
\end{aligned}
$$

Equations (4.28), (4.29), and (4.30) then combine to yield (4.27). q.e.d.

REMARK 4.14. For the sequel, we observe that the quantities $A, B, \widehat{R}$, and $\widehat{T}$ satisfy the following schematic equations:

$$
\begin{aligned}
D_{t} A & =R * A+\widehat{P} * \widehat{T}, \\
D_{t} B & =R * B+T * A+\widehat{P} * \nabla \widehat{T}+\widehat{P} * T * A, \\
\left(D_{t}-\Delta\right) \widehat{R}= & T * A+R * B+R * \widehat{R}+\widehat{P} * \bar{R}^{*} * \widehat{R}+\widehat{P} * R * \widehat{R}, \\
\left(D_{t}-\Delta\right) \widehat{T}= & \nabla T * A+R * B+R * \widehat{R}+\widehat{P} * \bar{R}^{*} * \widehat{R}+\widehat{P} * R * \widehat{R} \\
& +\widehat{P} * T * \widehat{R}+\widehat{P} * \bar{R}^{*} * \widehat{T}^{*} .
\end{aligned}
$$

For our purposes, the key feature of these equations is that each term contains at least one factor of (some contraction of) $A, B, \widehat{R}, \widehat{T}, \nabla \widehat{T}$, or their adjoints. Under our hypotheses, the other factors (including the extra linear factors of the components of our system) will be bounded, and this is enough for the application of the backwardsuniqueness result from $[\mathbf{K}]$, Theorem 5.1 , below.

4.7. Proof of Proposition 4.1. We are now in a position to prove Proposition 4.1. By the estimates of Shi $[\mathbf{S}]$, if $g(t)$ is a complete solution to (1.1) with

$$
|\operatorname{Rm}(x, t)|_{g(t)} \leq K_{0}
$$

on $M \times[0, T]$, then, for all $m \geq 1$, and all $\delta>0$, there exist constants $K_{m}=$ $K_{m}\left(n, K_{0}, T, \delta\right)$ such that

$$
\left|\nabla^{(m)} \operatorname{Rm}(x, t)\right|_{g(t)} \leq K_{m}
$$

on $M \times[\delta, T]$. The tensors $\bar{P}$ and $\widehat{P}$, being projection tensors, are also clearly bounded. In fact, if $\operatorname{dim} \mathcal{H}=k$, then $|\bar{P}|_{g(t)}^{2} \equiv k$ and $|\widehat{P}|_{g(t)}^{2} \equiv n(n-1) / 2-k$ on $M \times[0, T]$. Hence $\bar{R}, \widehat{R}, \bar{T}$, and $\widehat{T}$ (and their adjoints) are likewise uniformly bounded on $M \times[\delta, T]$. Thus we have only to verify that $A$ and $B$ are also bounded. This is more or less evident from the evolution equations (4.13), (4.14) at this point. We only need to observe first that, since $D_{t}=\frac{\partial}{\partial t}+R_{a b} \Lambda_{b}^{a}$, one has

$$
D_{t} U=\frac{\partial}{\partial t} U+\mathrm{Rc} * U
$$


for any tensor $U$ and then, that, from (4.35) and the above discussion, we have, on $M \times[\delta, T]$,

$$
\left|\frac{\partial}{\partial t} A\right| \leq C(|A|+1), \quad\left|\frac{\partial}{\partial t} B\right| \leq C(|A|+|B|+1),
$$

for an appropriate $C$. (Note that $\nabla \widehat{T}=A * T+\widehat{P} * \nabla T$.) At $t=T$, we have $|A|=|B|=0$, so we obtain that $|A|$ and (consequently) $|B|$ are bounded on $M \times[\delta, T]$ as well.

Taken with equations $(4.31)$ - (4.33), we have established that there exists a constant $C=C\left(n, K_{0}, T, \delta\right)$ such that

$$
\begin{aligned}
&\left|\frac{\partial}{\partial t} A\right|_{g(t)} \leq C\left(|A|_{g(t)}+|\widehat{T}|_{g(t)}\right), \\
&\left|\frac{\partial}{\partial t} B\right|_{g(t)} \leq C\left(|A|_{g(t)}+|B|_{g(t)}+|\nabla \widehat{T}|_{g(t)}\right), \\
&\left|\left(\frac{\partial}{\partial t}-\Delta\right) \widehat{R}\right|_{g(t)} \leq C\left(|A|_{g(t)}+|B|_{g(t)}+|\widehat{R}|_{g(t)}\right), \\
&\left|\left(\frac{\partial}{\partial t}-\Delta\right) \widehat{T}\right|_{g(t)} \leq C\left(|A|_{g(t)}+|B|_{g(t)}+|\widehat{R}|_{g(t)}+|\widehat{T}|_{g(t)}\right),
\end{aligned}
$$

on $M \times[\delta, T]$. Proposition 4.1 then follows at once from the Cauchy-Schwarz inequality.

\section{Backwards-uniqueness of the PDE-ODE system}

The following is a special case of Theorem 3.1 in $[\mathbf{K}]$.

Theorem 5.1. Let $\mathcal{X}$ and $\mathcal{Y}$ be finite direct sums of the bundles $T_{l}^{k}(M)$, and $X \in C^{\infty}(\mathcal{X} \times[A, \Omega]), Y \in C^{\infty}(\mathcal{Y} \times[A, \Omega])$. Suppose $g(t)$ is a smooth, complete solution to (1.1) of uniformly bounded curvature. Further assume that the sections $X, Y$, and $\nabla X$ are uniformly bounded with respect to $g(t)$ and satisfy

$$
\begin{aligned}
\left|\left(\frac{\partial}{\partial t}-\Delta_{g(t)}\right) X\right|_{g(t)}^{2} & \leq C\left(|X|_{g(t)}^{2}+|\nabla X|_{g(t)}^{2}+|Y|_{g(t)}^{2}\right), \\
\left|\frac{\partial Y}{\partial t}\right|_{g(t)}^{2} & \leq C\left(|X|_{g(t)}^{2}+|\nabla X|_{g(t)}^{2}+|Y|_{g(t)}^{2}\right)
\end{aligned}
$$

for some $C \geq 0$. Then $X(\cdot, \Omega) \equiv 0, Y(\cdot, \Omega) \equiv 0$ implies $X \equiv 0, Y \equiv 0$ on $M \times[A, \Omega]$.

Combining this result with Proposition 4.1, we have essentially proven Theorem 3.7 ; it only remains to see that the conclusion is valid all the way down to $t=0$.

Proof of Theorem 3.7. With $\mathcal{X}, \mathcal{Y}$, and $X(t), Y(t)$ defined as in the previous section, we may apply Proposition 4.1 and Theorem 5.1 on $M \times[\delta, T]$ for any $0<\delta<T$, to obtain the conclusion of Theorem 3.7 (and hence Theorem 1.4) for all $t \in(0, T)$. But $\bar{P}(t)$ and $\widehat{P}(t)$ are smoothly defined (and are complementary $g(t)$-orthogonal projections) on $\wedge^{2} T^{*} M$ for all $t \in[0, T]$. Thus the vanishing of $\nabla \bar{P}$ and $\nabla \widehat{P}$ on $M \times(0, T)$ imply by continuity that $\nabla \bar{P}(0)=\nabla \widehat{P}(0)=0$ also. Moreover, $\operatorname{ker}(\bar{P}(t)) \equiv$ $\mathcal{K}$ and $\operatorname{ker}(\widehat{P}(t)) \equiv \mathcal{H}$ for $t \in(0, T)$, thus continuity again implies that $\mathcal{K} \subset \operatorname{ker} \bar{P}(0)$ and $\mathcal{H} \subset$ ker $\widehat{P}(0)$. Since $\bar{P}(0)$ and $\widehat{P}(0)$ are complementary orthogonal projections, with

$$
\operatorname{rank} \bar{P}(0)=\operatorname{rank} \bar{P}(t) \equiv \operatorname{dim} \mathcal{H}, \quad \text { and } \quad \operatorname{rank} \widehat{P}(0)=\operatorname{rank} \widehat{P}(t) \equiv \operatorname{dim} \mathcal{K},
$$


we must actually have $\operatorname{ker}(\bar{P}(0))=\mathcal{K}$ and $\operatorname{ker}(\widehat{P}(0))=\mathcal{H}$. We also therefore have image $(\bar{P}(0))=\mathcal{H}$, and image $(\widehat{P}(0))=\mathcal{K}$, and it follows that $\mathcal{H}$ and $\mathcal{K}$ are orthogonal with respect to $g(0)$. Since $\bar{P}(0)$ and $\widehat{P}(0)$ are parallel, $\mathcal{H}$ and $\mathcal{K}$ are invariant under $\nabla_{g(0)}$-parallel translation by Lemma 3.4. Finally, since $(\operatorname{Rm} \circ \widehat{P})(t) \equiv 0$ for $0<t \leq T$, it follows that $\left.\operatorname{Rm}(0)\right|_{\mathcal{K}}: \mathcal{K} \rightarrow \wedge^{2} T^{*} M$ is also the zero map. The symmetry of $\mathrm{Rm}$ then implies that image $(\operatorname{Rm}(0)) \subset \mathcal{H}$ and, by Lemma 3.1, we conclude that $\mathfrak{h o l}_{p}(g(0)) \subset \mathcal{H}$, completing the proof.

q.e.d.

REMARK 5.2. By a result of S. Bando [B] (see also Remark 13.32 of [CCG2]), if $g(t)$ is a complete solution of (1.1) of bounded curvature, then $(M, g(t))$ is a realanalytic manifold for $0<t \leq T$. Hence at any $t>0$, any representative $\mathfrak{h o l}_{p}(g(t))$ of the isomorphism class of $\mathfrak{h o l}(g(t))$ is generated by the set

$$
\bigcup_{l=0}^{\infty}\left\{\nabla_{X_{1}} \nabla_{X_{2}} \cdots \nabla_{X_{l}} \operatorname{Rm}(p, t)(\omega) \mid X_{1}, X_{2}, \ldots, X_{l} \in T_{p} M, \quad \omega \in \wedge^{2} T_{p}^{*} M\right\} .
$$

(See $[\mathbf{K N}]$, Sections II.10, III.9.) Thus we can localize Theorem 1.4 somewhat: If, at some $p \in M$, the endomorphisms coming from the covariant derivatives of $\operatorname{Rm}(g(T))$ of all orders are contained in some subalgebra $H_{p} \subset \wedge^{2} T_{p}^{*} M$, then, at every $q, \mathfrak{h o l}_{q}(g(T))$ is contained in a subalgebra isomorphic to $H_{p}$. We can then apply Theorem 1.4 to conclude that, for all $(q, t) \in M \times[0, T], \mathfrak{h o l}_{q}(g(t))$ is contained a subalgebra isomorphic to $H_{p}$. In particular, if $g(T)$ admits a splitting on some neighborhood $U \subset M$ at some time $T>0, g(t)$ must split on a neighborhood of every $p \in M$ at all times $0 \leq t \leq T$.

\section{Appendix A. An alternative proof of the non-expansion of $\mathrm{Hol}^{0}(g(t))$}

In this section we present a second and essentially self-contained proof of Theorem 2.1, using the general framework of Theorem 1.1 (but different methods). Although we do not use the maximum principle for systems in [H2], the argument is close to that suggested by Hamilton for Theorem 4.1 of $[\mathbf{H 4}]$. We include it here only for reference and comparison purposes.

Theorem 2.1 has the following infinitesimal reformulation, corresponding to Theorem 1.4.

Claim. Suppose $\mathcal{H} \subset \wedge^{2} T^{*} M$ is a smooth subbundle that is invariant under $\nabla_{g(0)}$-parallel transportation and the bracket $[\cdot, \cdot]_{g(0)}$. If $\operatorname{Rm}(g(0)) \subset \mathcal{H}$, then it follows that $\operatorname{Rm}(g(t)) \subset \mathcal{H}$ for all $t$ and that $\mathcal{H}$ remains invariant by $\nabla_{g(t)}$-parallel transport and the bracket $[\cdot, \cdot]_{g(t)}$. In particular, $\mathfrak{h o l}_{p}(g(t)) \subset \mathcal{H}_{p}$.

Similar to the proof of Theorem 3.7, we extend the projection operators $\bar{P}_{0}$ and $\widehat{P}_{0}$ onto $\mathcal{H}$ and $\mathcal{K}=\mathcal{H}^{\perp}$ at time $t=0$ to operators $\bar{P}(t)$ and $\widehat{P}(t)$ for $t>0$. The key difference is that we accomplish this by the solution of a linear parabolic equation rather than by an ODE. Although with this choice we lose (temporarily) the assurance that the maps remain orthogonal projections, it allows us to effectively decouple our system and reduce the number of components from four to two.

Proof of the claim. Denote by $\mathcal{K} \subset \wedge^{2} T^{*} M$ the orthogonal complement of $\mathcal{H}$ and by $\bar{P}_{0}, \widehat{P}_{0}$ the projections onto $\mathcal{H}$ and $\mathcal{K}$ taken with respect to the metric induced by $g(0)$.

By assumption, $g(t)$ is complete and $\operatorname{Rm}(g(t))$ uniformly bounded, and so we can define $\bar{P}(t)$ and $\widehat{P}(t)$ on $M \times[0, T]$ to be the unique bounded solutions to the 
equations

$$
\begin{aligned}
& \left(\frac{\partial}{\partial t}-\Delta\right) \bar{P}_{a b c d}=-R_{a p} \bar{P}_{p b c d}-R_{b p} \bar{P}_{a p c d}-R_{c p} \bar{P}_{a b p d}-R_{d p} \bar{P}_{a b c p} \\
& \left(\frac{\partial}{\partial t}-\Delta\right) \widehat{P}_{a b c d}=-R_{a p} \widehat{P}_{p b c d}-R_{b p} \widehat{P}_{a p c d}-R_{c p} \widehat{P}_{a b p d}-R_{d p} \widehat{P}_{a b c p}
\end{aligned}
$$

with $\bar{P}(0)=\bar{P}_{0}$ and $\widehat{P}(0)=\widehat{P}_{0}$. As functions on $\widetilde{\mathcal{O}(M)}$, the above equations are

$$
\left(D_{t}-\Delta\right) \bar{P}_{a b c d}=0, \quad\left(D_{t}-\Delta\right) \widehat{P}_{a b c d}=0 .
$$

Since $\mathcal{H}$ is parallel initially, $A_{m i j k l} \doteqdot \nabla_{m} \widehat{P}_{i j k l} \equiv 0$ initially by Lemma 3.4. We claim $A_{m i j k l} \equiv 0$ for all $0 \leq t \leq T$. Its evolution is

$$
\begin{aligned}
\left(D_{t}-\Delta\right) A_{m i j k l} & =\left[\left(D_{t}-\Delta\right), \nabla_{m}\right] \widehat{P}_{i j k l} \\
& =2\left(R_{m b d c} \Lambda_{d}^{c} \nabla_{b}+R_{m c} \nabla_{c}\right) \widehat{P}_{i j k l},
\end{aligned}
$$

and so

$$
\left(\frac{\partial}{\partial t}-\Delta\right) A=R * A
$$

that is,

$$
\left|\left(\frac{\partial}{\partial t}-\Delta\right) A\right| \leq C|A|
$$

for $C=C(n, K)$. Defining $Q=|A|^{2}$, we thus have

$$
\begin{aligned}
\left(\frac{\partial}{\partial t}-\Delta\right) Q & =-2|\nabla \nabla \widehat{P}|^{2}+2\left\langle\left(\frac{\partial}{\partial t}-\Delta\right) A, A\right\rangle \\
& \leq 2 C Q
\end{aligned}
$$

SO

$$
Q(x, t) \leq e^{2 C T} \sup _{x \in M} Q(x, 0)=0
$$

on $M \times[0, T]$ by the maximum principle.

Strictly speaking, when $M$ is non-compact, our use of the maximum principle requires some justification. Since $M$ has bounded curvature (and, in particular, a lower bound on $\operatorname{Rc}(g(t))$ ), we need only to verify that $Q$ does not grow too quickly at infinity. We omit the full details of this verification, but point out that, for example, one could use a Bernstein-type trick, as in $[\mathbf{S}]$, and consider the quantity $F \doteqdot\left(L+|\widehat{P}|^{2}\right) Q$ where $L>0$ is constant. Then $F$ satisfies $F(p, 0) \equiv 0$, and, if $L=L\left(n, \sup |\widehat{P}|^{2}\right)$ is sufficiently large, the equation

$$
\left(\frac{\partial}{\partial t}-\Delta\right) F \leq C_{1} F-C_{2} F^{2}
$$

for positive constants $C_{i}=C_{i}\left(K_{0}, L, n\right)$. Using a standard cutoff function and the maximum principle, one can prove

$$
\sup _{B_{g(t)}(p, \rho) \times[0, T]} F(x, t) \leq C_{3}\left(n, K_{0}, L, T\right)\left(\frac{\rho+1}{\rho}\right)
$$

for all $\rho>>0$. Hence, upon sending $\rho \rightarrow \infty$, one obtains that $Q=|\nabla \widehat{P}|^{2} \leq C$ on $M \times[0, T]$.

We conclude, in any case, that $\widehat{P}$ remains parallel, and must actually satisfy the ODE $D_{t} \widehat{P}=0$. Likewise, we have $\nabla \bar{P}=0$ and $D_{t} \bar{P}=0$. But, by Lemmas 3.4 and 3.5 , this implies that $\bar{P}$ and $\widehat{P}$ remain complementary projections, and hence that $H(t) \doteqdot$ image $(\bar{P}(t))$ and $K(t) \doteqdot$ image $(\widehat{P}(t))$ remain complementary orthogonal $\nabla_{g(t)}$-parallel subbundles, with $H(t)$ invariant under the bracket $[\cdot, \cdot]_{g(t)}$. In particular $\mathcal{T}[\bar{P}, \widehat{P}, \bar{P}] \equiv 0$ by Lemma 4.4 . 
Now we define $\widehat{R}=\operatorname{Rm} \circ \widehat{P}$ as before. We have $\widehat{R}(0) \equiv 0$ by assumption, and claim $\widehat{R}(t) \equiv 0$ for all $0 \leq t \leq T$. Since $\nabla \widehat{P} \equiv 0$,

$$
\left(D_{t}-\Delta\right) \widehat{R}=\mathcal{Q}(\mathrm{Rm}) \circ \widehat{P} \text {. }
$$

Using $\mathcal{T}[\bar{P}, \widehat{P}, \bar{P}] \equiv 0$, we have, by (4.22) and Shi's estimates,

$$
|\mathcal{Q}(\mathrm{Rm}) \circ \widehat{P}|^{2} \leq C|\widehat{R}|^{2}
$$

So the (uniformly bounded) quantity $W=|\widehat{R}|^{2}$ satisfies

$$
\left(\frac{\partial}{\partial t}-\Delta\right) W \leq C W
$$

with $W(0) \equiv 0$; thus $W(t) \equiv 0$ by the maximum principle. Hence image $(\operatorname{Rm}(t)) \subset$ $H(t)$. Applying Proposition 3.6 shows that $H(t) \equiv \mathcal{H}$ and $K(t) \equiv \mathcal{K}$, and the theorem is proved.

q.e.d.

Acknowledgement. The author wishes to thank Professors Bennett Chow, Gerhard Huisken, and Lei Ni for their support and encouragement.

\section{References}

[A] Alexakis, Spyros. "Unique continuation for the vacuum Einstein equations." Feb 2009. 20pp. e-Print: arXiv:0902.1131.

[AS] Ambrose, W.; Singer, I. M. "A theorem on holonomy." Trans. Amer. Math. Soc. 75, (1953), $428-443$.

[B] Bando, Shigetoshi. "Real analyticity of solutions of Hamilton's equation." Math. Z. 195 (1987), no. 1, 93-97.

[Ber] Berger, Marcel. "Sur les groupes d'holonomie homogne des varits connexion affine et des varits riemanniennes." Bull. Soc. Math. France 83 (1955), 279-330.

[Bes] Besse, Arthur L. Einstein manifolds. (Reprint of the 1987 edition.) Classics in Mathematics. Springer-Verlag, Berlin, 2008. xii+516 pp.

[CCG2] Chow, Bennett; Chu, Sun-Chin; Glickenstein, David; Guenther, Christine; Isenberg, James; Ivey, Tom; Knopf, Dan; Lu, Peng; Luo, Feng; Ni, Lei The Ricci flow: techniques and applications. Part II. Analytic aspects. Mathematical Surveys and Monographs, 144. American Mathematical Society, Providence, RI, 2008. xxvi+458 pp.

[CLN] Chow, Bennett; Lu, Peng; Ni, Lei Hamilton's Ricci flow. Graduate Studies in Mathematics, 77. American Mathematical Society, Providence, RI; Science Press, New York, 2006. xxxvi+608 pp.

[CZ] Chen, Bing-Long; Zhu, Xi-Ping. "Uniqueness of the Ricci flow on complete noncompact manifolds." J. Differential Geom. 74 (2006), no. 1, 119-154.

[DR] De Rham, Georges. "Sur la reductibilit d'un espace de Riemann." Comment. Math. Helv. 26, (1952). 328344 .

[DT] De Turck, Dennis M. "Deforming metrics in the direction of their Ricci tensors." J. Differential Geom. 18 (1983), no. 1, 157-162.

[H1] Hamilton, Richard S. "Three-manifolds with positive Ricci curvature." J. Differential Geom. 17 (1982), no. 2, 255-306.

[H2] Hamilton, Richard S. "Four-manifolds with positive curvature operator." J. Differential Geom. 24 (1986), no. 2, 153-179.

[H3] Hamilton, Richard S. "The Harnack estimate for the Ricci flow." J. Differential Geom. 37 (1993), no. $1,225-243$.

[H4] Hamilton, Richard S. "The formation of singularities in the Ricci flow." Surveys in differential geometry, Vol. II (Cambridge, MA, 1993), 7136, Int. Press, Cambridge, MA, 1995.

[J] Joyce, Dominic D. Riemannian holonomy groups and calibrated geometry. Oxford Graduate Texts in Mathematics, 12. Oxford University Press, Oxford, 2007. x+303 pp.

[K] Kotschwar, Brett L. "Backwards uniqueness for the Ricci flow." Int. Math. Res. Not. (2010), no. 21, $4064-4097$.

[KN] Kobayashi, Shoshichi; Nomizu, Katsumi. Foundations of differential geometry: Vol I. Interscience Publishers, New York-London, 1963. xi+329 pp.

[KW] Kazdan, Jerry L.; Warner, F. W. "Existence and conformal deformation of metrics with prescribed Gaussian and scalar curvatures." Ann. of Math. (2) 101 (1975), 317331. 
[S] Shi, Wan-Xiong. "Deforming the metric on complete Riemannian manifolds." J. Differential Geom. 30 (1989), no. 1, 223-301.

Max Planck Institute for Gravitational Physics, Am Mühlenberg 1, D-14476 Golm, Germany

E-mail address: brett.kotschwar@aei.mpg.de 